\title{
Minkowski, Schwarzschild and Kerr Metrics Revisited
}

\author{
J.-F. Pommaret \\ CERMICS, Ecole des Ponts ParisTech, Paris, France \\ Email: jean-francois.pommaret@wanadoo.fr
}

How to cite this paper: Pommaret, J.-F. (2018) Minkowski, Schwarzschild and Kerr Metrics Revisited. Journal of Modern Physics, 9, 1970-2007.

https://doi.org/10.4236/jmp.2018.910125

Received: June 19, 2018

Accepted: September 10, 2018

Published: September 13, 2018

Copyright $\odot 2018$ by author and Scientific Research Publishing Inc. This work is licensed under the Creative Commons Attribution International License (CC BY 4.0).

http://creativecommons.org/licenses/by/4.0/

\begin{abstract}
In recent papers, a few physicists studying Black Hole perturbation theory in General Relativity (GR) have tried to construct the initial part of a differential sequence based on the Kerr metric, using methods similar to the ones they already used for studying the Schwarzschild geometry. Of course, such a differential sequence is well known for the Minkowski metric and successively contains the Killing (order 1), the Riemann (order 2) and the Bianchi (order 1 again) operators in the linearized framework, as a particular case of the Vessiot structure equations. In all these cases, they discovered that the compatibility conditions (CC) for the corresponding Killing operator were involving a mixture of both second order and third order CC and their idea has been to exhibit only a minimal number of generating ones. Unhappily, these physicists are neither familiar with the formal theory of systems of partial differential equations and differential modules, nor with the formal theory of Lie pseudogroups. Hence, even if they discovered a link between these differential sequences and the number of parameters of the Lie group preserving the background metric, they have been unable to provide an intrinsic explanation of this fact, being limited by the technical use of Weyl spinors, complex Teukolsky scalars or Killing-Yano tensors. The purpose of this difficult computational paper is to provide differential and homological methods in order to revisit and solve these questions, not only in the previous cases but also in the specific case of any Lie group or Lie pseudogroup of transformations. These new tools, which are now available as computer algebra packages, question the mathematical foundations of GR and the origin of gravitational waves.
\end{abstract}

\section{Keywords}

General Relativity, Killing Operator, Riemann Tensor, Weyl Tensor, Bianchi Identities, Lie Algebroid, Differential Sequence, Differential Module, Homological Algebra, Extension Modules 


\section{Introduction}

In many recent technical papers, a few physicists working on General Relativity (GR) are trying to construct high order differential sequences while starting with the Killing operator for a given metric (Minkowski, Schwarzschild, Kerr, ...) ([1] [2] [3] [4] [5]). The "ad hoc" (technical) methods involved are ranging from Killing/Killing-Yano tensors, Penrose spinors, Teukolski scalars or compexified frames.

Meanwhile, on one side, they have the feeling that the construction of these sequences has surely to do with the fact that the corresponding Killing systems of infinitesimal Lie equations have less linearly independent solutions than the $n(n+1) / 2$ that were predicted by A. Eisenhart in 1949 for nondegenerate metrics with constant Riemannian curvature on a space of dimension $n$ ([6] along results first found by E. Vessiot in 1903 [7] [8]), that is 10 when $n=4$ (space-time) with the Minkowski metric, and discover that, perhaps for this reason, they have to exhibit an unexpected mixture of generating compatibility conditions (CC) of order 2 and 3.

However, on another side, they are clearly aware of the fact that their results are far from being intrinsic and cannot be adapted to other metrics or dimensions.

The purpose of this short but difficult paper, even though it is written in a rather self-contained computational way, is to solve this problem in its full intrinsic generality, using a few results ranging among the most delicate ones that can be found in the formal theory of systems of ordinary differential (OD) or partial differential (PD) equations and Lie pseudogroups introduced around 1970 by D. C. Spencer and coworkers ([9] [10] [11]). It must also be noticed that these new methods, though they can be found in many books ([12]-[18]) or papers ([19]-[25]) of the author of this paper to which we shall often refer, have rarely been acknowledged by the computer algebra community and/or by physicists. The essential new "trick" is to deal with sections of jet-bundles and not with solutions of systems of OD or PD equations.

In Section 2, we provide and illustrate the two crucial mathematical results needed for the applications presented in Section 3. The first result, roughly discovered by M. Janet in 1920 ([26]), describes the way to use a certain prolongation/projection ( $\mathrm{PP}$ ) procedure absolutely needed in order to transform any sufficiently regular system into a formally integrable system and, finally, even an involutive system, that is a situation where we know that the generating CC are described by a first order involutive system and the possibility to construct a canonical Janet or Spencer sequence. In this homological algebraic framework, the technique of diagram chasing is absolutely needed and we ask the reader to learn at least the so-called "snake" lemma in textbooks ([27]-[32]). As for the second result, it is allowing to study effectively Lie operators acting on vector fields and such that, if two vectors are solutions, their bracket is also a solution. It is thus also absolutely needed when dealing with the PP procedure 
because it is not possible to work out solutions in general and the use of computer algebra is therefore a pure nonsense for the intrinsic study of most Lie operators as we shall see in Section 3.

The author thanks Prof. Lars Andersson from Albert Einstein Institute of Potsdam for many enlightening discussions during his last visit to AEI (October 23-27, 2017), in particular for bringing the problem of finding new generating CC to his attention when studying the specific Lie operators to be met in GR.

\section{Mathematical Tools}

\section{A) Formal Integrability}

All operators and systems considered in this paper will have coefficients in a differential field $K$ with $n$ derivations $\partial_{1}, \cdots, \partial_{n}$, for example the standard derivations when $K=\mathbb{Q}\left(x^{1}, \cdots, x^{n}\right)$ is the differential field of rational functions. Starting with a given operator $\mathcal{D}$, a direct problem is to look for generating compatibility conditions (CC) described by an operator $\mathcal{D}_{1}$ in such a way that $\mathcal{D}_{1} \eta=0$ denotes the CC needed for solving (at least locally) the linear inhomogeneous system $\mathcal{D} \xi=\eta$. Going along this way, one may construct inductively a differential sequence $\mathcal{D}, \mathcal{D}_{1}, \mathcal{D}_{2}, \cdots$ of operators such that not only $\mathcal{D}_{i+1} \circ \mathcal{D}_{i}=0$ of course but also each operator $\mathcal{D}_{i+1}$ generates the CC of $\mathcal{D}_{i}$. Such a result has been found for the first time as a footnote by M. Janet in 1920 ([26]) who even proved that, under slightly additional conditions, the sequence stops at $\mathcal{D}_{n}$ which is thus formally surjective, when $n$ is the number of independent variables.

The main problem is that, in general and though surprising it may look like, the word "generating" has no clear meaning at all, a result leading to refine the definition of a differential sequence in an intrinsic way. Apart from the Macaulay example that will be treated and revisited later on, our two favorite examples are the following ones that will also be revisited and are treated in a way adapted to the aim of this paper. We shall denote by $m$ the number of unknowns $\left(y^{1}, \cdots, y^{m}\right)$ also called differential indeterminates, by $n$ the number of independent variables $\left(x^{1}, \cdots, x^{n}\right)$ and by $q$ the order of the system/operator considered. We shall finally introduce the non-commutative ring $D=K[d]=K\left[d_{1}, \cdots, d_{n}\right]$ of differential operators $P, Q, \cdots$ with coefficients in $K$.

EXAMPLE 2A.1: With $m=1, n=2, K=\mathbb{Q}$, we shall use formal derivatives $\left(d_{1}, d_{2}\right)$ when using differential modules or computer algebra but will set $d_{i}\left(d_{j} y\right)=d_{j}\left(d_{i} y\right)=d_{i j} y=y_{i j}$ for simplicity while using jet coordinates and notations. Let us consider the second order system:

$$
P y \equiv y_{22}=u, Q y \equiv y_{12}+y=v \Rightarrow y_{2}=v_{2}-u_{1} \Rightarrow y=v-v_{12}+u_{11}
$$

There are many different ways to look at such a system. The first natural one is to say that the only solution is $y=0$ when $u=v=0$. The second one is to look for the CC that must be satisfied by $(u, v)$ and we may adopt two possible presentations: 
- Substitute $y$ and obtain the 2 fourth order CC:

$$
\left\{\begin{array}{l}
A \equiv v_{1222}-v_{22}-u_{1122}+u=0 \\
B \equiv v_{1122}-u_{1112}-u_{11}=0
\end{array}\right.
$$

which are not differentially independent because one can easily check:

$$
B_{12}-B-A_{11}=0
$$

- However, we also have:

$$
P \circ Q-Q \circ P=0 \Rightarrow C \equiv P v-Q u \equiv v_{22}-u_{12}-u=0
$$

that is a second order CC.

Finally, we obtain:

$$
A \equiv C_{12}-C, B \equiv C_{11} \Leftrightarrow C \equiv B_{22}-A_{12}+A
$$

and we discover that the $\mathrm{CC}$ of $\mathcal{D}=(P, Q)$ are generated by $(A, B)$ but also by $C$ alone, though any student will hesitate between the two possibilities!.

Referring to differential homological algebra as in ([25]) while indicating the order of an operator below its arrow, the same trivial differential module $M=0$ (care) defined by $\mathcal{D}$ has therefore two split resolutions:

$$
\begin{gathered}
0 \rightarrow D \underset{2}{\longrightarrow} D^{2} \underset{4}{\longrightarrow} D^{2} \underset{2}{\longrightarrow} D \rightarrow 0 \\
0 \rightarrow D \underset{2}{\longrightarrow} D^{2} \underset{2}{\longrightarrow} \rightarrow 0
\end{gathered}
$$

which are quite different even if the two Euler-Poincaré characteristics both vanish because we have:

$$
1-2+2-1=0, \quad 1-2+1=0
$$

EXAMPLE 2A.2: (Janet) Now with $m=1, n=3, q=2$ and $K=\mathbb{Q}\left(x^{2}\right)$, let us consider the second order system (see [15] [16] for more details):

$$
y_{33}-x^{2} y_{11}=u, \quad y_{22}=v
$$

We let the reader use computer algebra and Gröbner bases in order to find out the two generating $\mathrm{CC}$ of respective order 3 and 6 and work out the following possible resolution where $\operatorname{dim}_{K}(M)=12$ :

$$
0 \rightarrow D \rightarrow D^{2} \underset{6}{\longrightarrow} D^{2} \underset{2}{\longrightarrow} \rightarrow M \rightarrow 0 \text {. }
$$

The main point in this subsection is to use the three following highly non-trivial theorems (Compare [10] [11] to [12] [16], in particular pp. 364-366 for details) and just follow their proofs on the two previous examples but the next example found by Macaulay will provide all details.

When $X$ is a manifold with $\operatorname{dim}(X)=n$ and local coordinates $\left(x^{1}, \cdots, x^{n}\right)$, we denote by $T$ the tangent bundle and by $T^{*}$ the cotangent bundle. If $E$ is a vector bundle over $X$ with projection $\pi$, local coordinates $\left(x, y^{k}\right)$ for $k=1, \cdots, m$, we use to denote by $\operatorname{dim}(E)=m$ the fiber dimension of $E$. A local or global section $f$ can be locally described by $y^{k}=f^{k}(x)$. Using multi-indices $\mu=\left(\mu_{1}, \cdots, \mu_{n}\right)$ with length $|\mu|=\mu_{1}+\cdots+\mu_{n}$, we shall denote by $J_{q}(E)$ the $q$-jet bundle of $E$ over $X$ with projection $\pi_{q}$ on $X$, local coordinates $x^{i}, y_{\mu}^{k}$ 
and sections $f_{q}=\left(f_{\mu}^{k}(x)\right)$ over $f$ or $j_{q}(f)=\left(\partial_{\mu} f^{k}(x)\right)$. The Spencer operator $D: J_{q+1}(E) \rightarrow T^{*} \otimes J_{q}(E): f_{q+1} \rightarrow j_{1}\left(f_{q}\right)-f_{q+1}$ $=\left(\partial_{i} f^{k}(x)-f_{i}^{k}(x), \partial_{i} f_{j}^{k}(x)-f_{i j}^{k}(x), \cdots\right)$

(care to the notation) will allow

to distinguish among these two types of sections. We denote by $S_{q} T^{*}$ the vector bundle of (completely) symmetric tensors and by $\wedge^{r} T^{*}$ the vector bundle of (completely) skewsymmetric tensors over $X$. We set:

DEFINITION 2A.3: A system of order $q$ on $E$ is an open vector subbundle $R_{q} \subseteq J_{q}(E)$ with prolongations

$\rho_{r}\left(R_{q}\right)=R_{q+r}=J_{r}\left(R_{q}\right) \cap J_{q+r}(E) \subseteq J_{r}\left(J_{q}(E)\right)$ and symbols $\rho_{r}\left(g_{q}\right)=g_{q+r}=S_{q+r} T^{*} \otimes E \bigcap R_{q+r} \subseteq J_{q+r}(E)$ only depending on

$g_{q} \subseteq S_{q} T^{*} \otimes E$. For $r, s \geq 0$, we denote by $R_{q+r}^{(s)} \subseteq R_{q+r}=\pi_{q+r}^{q+r+s}\left(R_{q+r+s}\right)$ the projection of $R_{q+r+s}$ on $R_{q+r}$, which is thus defined by more equations in general. The system $R_{q}$ is said to be formally integrable (FI) if we have $R_{q+r}^{(s)}=R_{q+r}, \forall r, s \geq 0$, that is if all the equations of order $q+r$ can be obtained by means of only $r$ prolongations. The system $R_{q}$ is said to be involutive if it is FI with an involutive symbol $g_{q}$. We shall simply denote by $\Theta=\left\{f \in E \mid j_{q}(f) \in R_{q}\right\}$ the "set" of (formal) solutions. It is finally easy to prove that the Spencer operator restricts to $D: R_{q+1} \rightarrow T^{*} \otimes R_{q}$.

Comparing the sequences obtained in the two previous examples, we may state:

DEFINITION 2A.4: A differential sequence is said to be formally exact if it is exact on the jet level composition of the prolongations involved. A formally exact sequence is said to be strictly exact if all the operators/systems involved are FI. A strictly exact sequence is called canonical if all the operators/systems are involutive. The only known canonical sequences are the Janet and Spencer sequences that can be defined independently from each other.

EXAMPLE 2A.5: In the first example with $\operatorname{dim}(E)=1, \operatorname{dim}\left(F_{0}\right)=2$, we let the reader prove that $\operatorname{dim}\left(R_{2+r}\right)=4, \forall r \geq 0$. Hence, if $(A, B)$ is a section of $F_{1}$ while $C$ is a section of $F_{1}^{\prime}$, the jet prolongation sequence:

$$
\begin{aligned}
0 \rightarrow R_{6} & \rightarrow J_{6}(E) \rightarrow J_{4}\left(F_{0}\right) \rightarrow F_{1} \rightarrow 0 \\
0 & \rightarrow 4 \rightarrow 28 \rightarrow 30 \rightarrow 2 \rightarrow 0
\end{aligned}
$$

is not formally exact because $4-28+30-2=4 \neq 0$, while the corresponding long sequence:

$$
\begin{gathered}
0 \rightarrow R_{r+4} \rightarrow J_{r+4}(E) \rightarrow J_{r+2}\left(F_{0}\right) \rightarrow J_{r}\left(F_{1}^{\prime}\right) \rightarrow 0 \\
0 \rightarrow 4 \rightarrow(r+5)(r+6) / 2 \rightarrow(r+3)(r+4) \rightarrow(r+1)(r+2) / 2 \rightarrow 0
\end{gathered}
$$

is indeed formally exact because

$4-\frac{\left(r^{2}+11 r+30\right)}{2}+\left(r^{2}+7 r+12\right)-\frac{\left(r^{2}+3 r+2\right)}{2}=0$ but not strictly exact because $R_{2}$ is quite far from being FI.

With canonical projection $\Phi_{0}: J_{q}(E) \Rightarrow J_{q}(E) / R_{q}=F_{0}$, the case $r=0, s=1$ is described by the following commutative and exact diagram: 


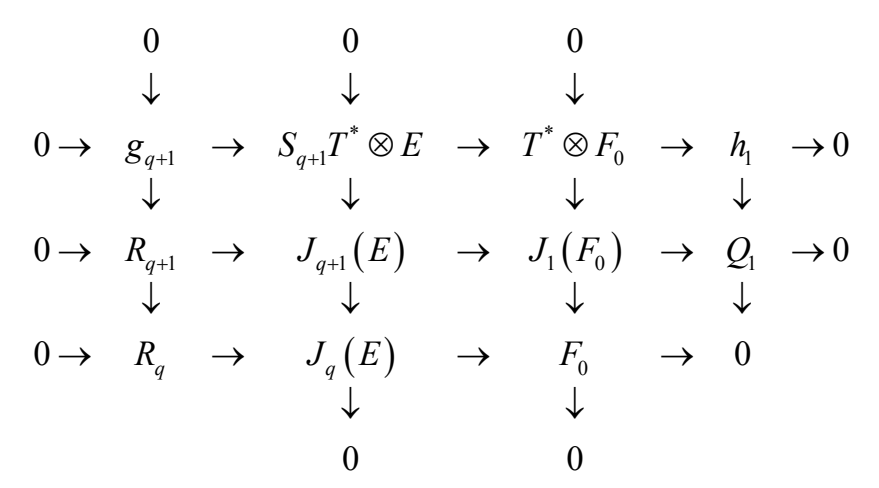

Chasing in this diagram while applying the "snake" lemma, we obtain the useful long exact connecting sequence:

$$
0 \rightarrow g_{q+1} \rightarrow R_{q+1} \rightarrow R_{q} \rightarrow h_{1} \rightarrow Q_{1} \rightarrow 0
$$

which is thus connecting in a tricky way FI (lower left) with CC (upper right).

Having in mind the fact that the Riemann ( $g_{1}$ is not 2-acyclics but $g_{2}=2$ is trivially involutive) and the Weyl ( $\hat{g}_{1}$ is not 2 -acyclic but $\hat{g}_{2}$ is 2 -acyclic $\forall n \geq 4$ while $\hat{g}_{3}=0, \forall n \geq 3$ ) operators (linearization of the respective tensors) are second order, a key stone is:

THEOREM 2A.6: If a system $R_{q} \subset J_{q}(E)$ is FI, then the corresponding operator $\mathcal{D}: E \stackrel{j_{q}}{\longrightarrow} J_{q}(E) \stackrel{\Phi_{0}}{\longrightarrow} J_{q}(E) / R_{q}=F_{0}$ admits (minimal) generating CC of order $s+1$ if $s$ is the smallest integer such that $g_{q+s}$ becomes 2-acyclic.

THEOREM 2A.7: (prolongation/projection (PP) procedure) If a system $R_{q} \subseteq J_{q}(E)$ has a 2 -acyclic symbol $g_{q} \subseteq S_{q} T^{*} \otimes E$ and $g_{q+1}$ is a vector bundle, then $\rho_{r}\left(R_{q}^{(1)}\right)=R_{q+r}^{(1)}, \forall r \geq 0$. Hence, if an arbitrary system $R_{q} \subseteq J_{q}(E)$ is given, one can effectively find two integers $r, s \geq 0$ such that the system $R_{q+r}^{(s)}$ is formally integrable or even involutive, with the same solutions.

THEOREM 2A.8: Starting with anyoperator $\mathcal{D}=E \rightarrow F_{0}$ of order $q$ defined over a differential field $K$ by a system $R_{q} \subseteq J_{q}(E)$, one can construct a formally exact commutative diagram:

$$
\begin{array}{rllllllll}
0 \rightarrow & \Theta & \rightarrow & E & \stackrel{\mathcal{D}}{\rightarrow} & F_{0} & \stackrel{\mathcal{D}_{1}}{\rightarrow} & F_{1} \\
& \| & & \| & & \downarrow \mathcal{P} & & \downarrow \mathcal{Q} \\
0 \rightarrow & \Theta & \rightarrow & E & \stackrel{\mathcal{D}^{\prime}}{\rightarrow} & F_{0}^{\prime} & \stackrel{\mathcal{D}_{1}^{\prime}}{\rightarrow} & F_{1}^{\prime}
\end{array}
$$

where $\mathcal{P}$ is an injective operator of order at least $r+s$ whenever $R_{q+r}^{(s)}$ is a formally integrable system with the same solutions as $R_{q}$ obtained by the PP procedure and the upper sequence is formally exact while the lower sequence is strictly exact with $\operatorname{ord}\left(\mathcal{D}_{1}^{\prime}\right)=1$ when $\mathcal{D}^{\prime}$ is involutive.

Starting with an arbitrary system $R_{q} \subset J_{q}(E)$, the main purpose of the next crucial example is to prove that the generating CC of the operator $\mathcal{D}=\Phi_{0} \circ j_{q}: E \stackrel{j_{q}}{\longrightarrow} J_{q}(E) \stackrel{\Phi_{0}}{\longrightarrow} J_{q}(E) / R_{q}=F_{0}$, though they are of course fully determined by the first order CC of the final involutive system $R_{q+r}^{(s)}$ produced by the up/down PP procedure, are in general of order $r+s+1$ as we 
shall see in the applications but may be of strictly lower order. All diagrams and chases will be described in actual practice. We invite the reader to study similarly the first example where $R_{2}^{(4)}=0, \mathcal{D}^{\prime}=j_{2}$ and $\mathcal{D}_{1}^{\prime}=D_{1}$ is the first Spencer operator.

EXAMPLE 2A.9: ([33], p 40 with the first feeling of Formal Integrability). With $m=1, n=3, q=2$, let us consider the second order linear system $R_{2} \subset J_{2}(E)$ with $\operatorname{dim}(E)=1, \operatorname{dim}\left(R_{2}\right)=8$ and par $_{2}=\left\{y, y_{1}, y_{2}, y_{3}, y_{11}, y_{12}, y_{22}, y_{23}\right\}$ if we use jet coordinates, defined by the two inhomogeneous PD equations:

$$
P y \equiv y_{33}=u, \quad Q y \equiv y_{13}+y_{2}=v
$$

As we already said, the only existing intrinsic procedure has two steps:

- Step 1: First of all we have to look for the symbol $g_{2}$ defined by the two linear equations $v_{33}=0, v_{13}=0$. The coordinate system is not $\delta$-regular and exchanging $x^{1}$ with $x^{2}$, we get the Janet board:

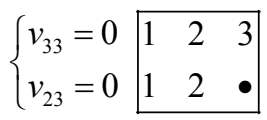

It follows that $g_{2}$ is involutive, thus 2 -acyclic and we obtain from the main theorem $\rho_{r}\left(R_{2}^{(1)}\right)=R_{2+r}^{(1)}$. However, $R_{2}^{(1)} \subset R_{2}$ with a strict inclusion because $R_{2}^{(1)}$ with $\operatorname{dim}\left(R_{2}^{(1)}\right)=7$ is now defined by the 3 equations:

$$
y_{33}=u, \quad y_{23}=v_{3}-u_{1}, \quad y_{13}+y_{2}=v
$$

We may start again with $R_{2}^{(1)}$ and study its symbol $g_{2}^{(1)}$ defined by the 3 linear equations with Janet board:

$$
\left\{\begin{array}{l}
v_{33}=0 \\
v_{23}=0 \\
v_{13}=0
\end{array}\left[\begin{array}{lll}
1 & 2 & 3 \\
1 & 2 & \bullet \\
1 & \bullet & \bullet
\end{array}\right]\right.
$$

It follows that $g_{2}^{(1)}$ is again involutive but we have a strict inclusion $R_{2}^{(2)} \subset R_{2}^{(1)}$ because $\operatorname{dim}\left(R_{2}^{(2)}\right)=6$ as $R_{2}^{(2)}$ is defined by the 4 equations with Janet board:

$$
\left\{\begin{array}{l}
y_{33}=u \\
y_{23}=v_{3}-u_{1} \\
y_{22}=v_{2}-v_{13}+u_{11} \\
y_{13}-y_{2}=v
\end{array}\left[\begin{array}{lll}
1 & 2 & 3 \\
1 & 2 & \bullet \\
1 & 2 & \bullet \\
1 & \bullet & \bullet
\end{array}\right]\right.
$$

As $R_{2}^{(2)}$ is easily seen to be involutive, we achieve the PP procedure with the strict intrinsic inclusions and corresponding fiber dimensions:

$$
R_{2}^{(2)} \subset R_{2}^{(1)} \subset R_{2} \Leftrightarrow 6<7<8
$$

Finally, we have $\rho_{r}\left(R_{2}^{(2)}\right)=\rho_{r}\left(\left(R_{2}^{(1)}\right)^{(1)}\right)=\left(\rho_{r}\left(R_{2}^{(1)}\right)\right)^{(1)}=\left(R_{2+r}^{(1)}\right)^{(1)}=R_{2+r}^{(2)}$.

- Step 2: It remains to find out the CC for $(u, v)$ in the initial inhomogeneous system. As we have used two prolongations in order to exhibit $R_{2}^{(2)}$, we have second order formal derivatives of $u$ and $v$ in the right members. Now, as we 
have an involutive system, we have first order CC for the new right members and could hope therefore for third order generating CC. However, we have successively the 4 CC:

$$
\begin{gathered}
y_{233}=d_{3}\left(v_{3}-u_{1}\right)=d_{2} u \Rightarrow v_{33}-u_{13}-u_{2}=0 \\
y_{223}=d_{3}\left(v_{2}-v_{13}+u_{11}\right)=d_{2}\left(v_{3}-u_{1}\right) \Rightarrow v_{133}-u_{113}-u_{12}=0 \\
y_{133}+y_{23}=d_{3} v=d_{1} u+\left(v_{3}-u_{1}\right) \Rightarrow 0=0 \\
y_{123}+y_{22}=d_{2} v=d_{1}\left(v_{3}-u_{1}\right)+\left(v_{2}-v_{13}+u_{11}\right) \Rightarrow 0=0
\end{gathered}
$$

It follows that we have only one second and one third order CC:

$$
v_{33}-u_{13}-u_{2}=0, \quad v_{133}-u_{113}-u_{12}=0
$$

but, surprisingly, the only generating second order CC $v_{33}-u_{13}-u_{2}=0$ which is coming from the fact that the operator $P$ commutes with the operator $Q$.

We finally show how FI is related to CC by means of an homological procedure known as the "long exact connecting sequence" which is the main byproduct of the so-called snake lemma used when chasing in diagrams. Needless to say that absolutely no classical procedure can produce such a result which is thus totally absent from the GR papers already quoted. First of all, let us compute the dimensions of the bundles that will be used in the following diagrams while using parametric jets:

$$
\begin{gathered}
\operatorname{par}_{2}=\left\{y, y_{1}, y_{2}, y_{3}, y_{11}, y_{12}, y_{22}, y_{23}\right\} \\
\operatorname{par}_{3}=\left\{y, y_{1}, y_{2}, y_{3}, y_{11}, y_{12}, y_{22}, y_{111}, y_{112}, y_{122}, y_{222}, y_{223}\right\} \\
\operatorname{par}_{4}=\left\{y, y_{1}, y_{2}, y_{3}, y_{11}, y_{12}, y_{111}, y_{112}, y_{122}, y_{222}, y_{1111}, y_{1112}, y_{1122}, y_{1222}, y_{2222}, y_{2223}\right\}
\end{gathered}
$$

and thus $\operatorname{dim}\left(R_{2}\right)=8, \operatorname{dim}\left(R_{3}\right)=12, \operatorname{dim}\left(R_{4}\right)=16 \quad$ in the following commutative and exact diagram where $E$ is the trivial vector bundle with $\operatorname{dim}(E)=1$ and $\operatorname{dim}\left(g_{2+r}\right)=4+r$ :

$$
\begin{aligned}
& 0 \quad 0 \quad 0 \\
& \downarrow \quad \downarrow \quad \downarrow
\end{aligned}
$$

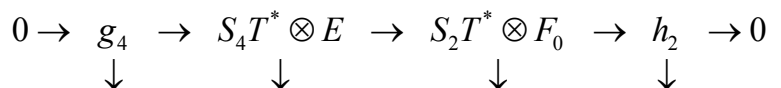

$$
\begin{aligned}
& 0 \rightarrow R_{4} \rightarrow J_{4}(E) \rightarrow J_{2}\left(F_{0}\right) \rightarrow F_{1} \rightarrow 0 \\
& 0 \rightarrow R_{3} \rightarrow J_{3}(E) \rightarrow J_{1}\left(F_{0}\right) \rightarrow 0 \\
& \downarrow \quad \downarrow \\
& 0 \quad 0
\end{aligned}
$$

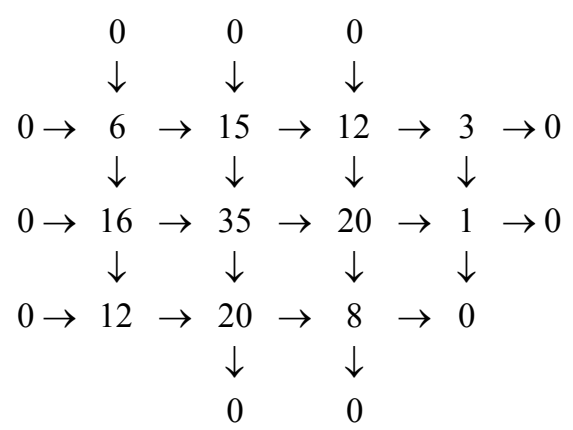




$$
\begin{aligned}
& \begin{array}{cccc}
0 & 0 & 0 & 0 \\
\downarrow & \downarrow & \downarrow & \downarrow
\end{array} \\
& 0 \rightarrow \quad g_{4} \quad \rightarrow \quad S_{4} T^{*} \otimes E \quad \rightarrow \quad S_{2} T^{*} \otimes F_{0} \quad \rightarrow \quad h_{2} \quad \rightarrow 0 \\
& \downarrow \delta \quad \downarrow \delta \quad \downarrow \delta \quad \downarrow \delta \\
& 0 \rightarrow \quad T^{*} \otimes g_{3} \quad \rightarrow \quad T^{*} \otimes S_{3} T^{*} \otimes E \quad \rightarrow \quad T^{*} \otimes T^{*} \otimes F_{0} \quad \rightarrow \quad T^{*} \otimes h_{1} \quad \rightarrow 0 \\
& \downarrow \delta \quad \downarrow \delta \quad \downarrow \delta \quad \downarrow \\
& 0 \rightarrow \quad \wedge^{2} T^{*} \otimes g_{2} \quad \rightarrow \quad \wedge^{2} T^{*} \otimes S_{2} T^{*} \otimes E \quad \rightarrow \quad \wedge^{2} T^{*} \otimes F_{0} \quad \rightarrow \quad 0 \\
& \downarrow \delta \quad \downarrow \delta \quad \downarrow \\
& 0 \rightarrow \wedge^{3} T^{*} \otimes T^{*} \otimes E \quad=\quad \wedge^{3} T^{*} \otimes T^{*} \otimes E \quad \rightarrow \quad 0 \\
& \downarrow \\
& 0
\end{aligned}
$$

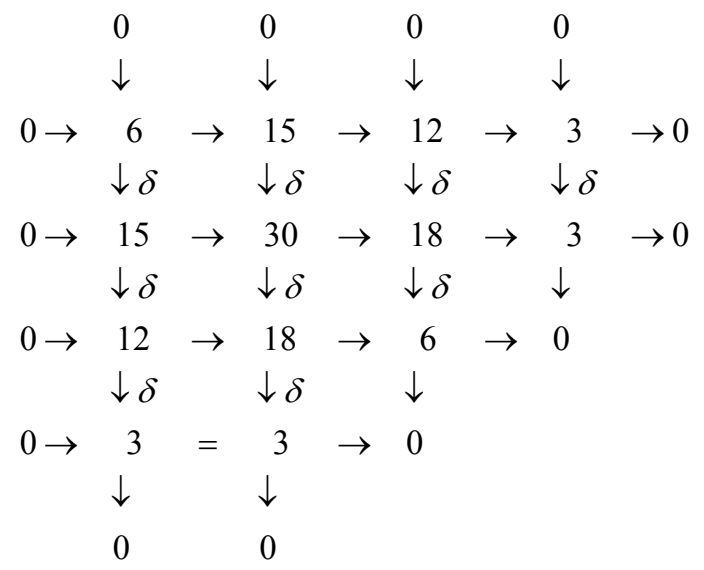

where $S_{4} T^{*} \otimes E \simeq S_{4} T^{*}$ and $F_{1} \simeq Q_{2}$. From the snake lemma and a chase, we obtain the long exact connecting sequence.

$$
\begin{gathered}
0 \rightarrow g_{4} \rightarrow R_{4} \rightarrow R_{3} \rightarrow h_{1} \rightarrow F_{1} \rightarrow 0 \\
0 \rightarrow 6 \rightarrow 16 \rightarrow 12 \rightarrow 3 \rightarrow 1 \rightarrow 0
\end{gathered}
$$

relating FI (lower left) to CC (upper right). By composing the epimorphism $S_{2} T^{*} \otimes F_{0} \rightarrow h_{1}$ with the epimorphism $h_{1} \rightarrow F_{1}$, we obtain an epimorphism $S_{2} T^{*} \otimes F_{0} \rightarrow F_{1}$ and the long exact sequence:

$$
0 \rightarrow g_{4} \rightarrow S_{4} T^{*} \otimes E \rightarrow S_{2} T^{*} \otimes F_{0} \rightarrow F_{1} \rightarrow 0
$$

which is nevertheless not a long ker/coker exact sequence by counting the dimensions as we have $6-15+12-1=2 \neq 0$. In order to convince the reader about the usefulness of these new methods, even on such an elementary example, let us prove directly the exactness of the following long exact sequence $\forall r \geq 0$ :

$$
0 \rightarrow R_{r+4} \rightarrow J_{r+4}(E) \rightarrow J_{r+2}\left(F_{0}\right) \rightarrow J_{r}\left(F_{1}\right) \rightarrow 0
$$

We let the reader check that $\operatorname{dim}\left(R_{r+2}\right)=4 r+8, \forall r \geq 0$ and thus $\operatorname{dim}\left(R_{r+4}\right)=4 r+16, \forall r \geq 0$ as a tricky exercise of combinatorics and then use the standard formulas:

$$
\begin{aligned}
& \operatorname{dim}\left(J_{r+4}(E)\right)=(r+5)(r+6)(r+7) / 6 \\
& \operatorname{dim}\left(J_{r+2}\left(F_{0}\right)\right)=(r+3)(r+4)(r+5) / 3
\end{aligned}
$$




$$
\operatorname{dim}\left(J_{r}\left(F_{1}\right)\right)=(r+1)(r+2)(r+3) / 6
$$

in order to check that the Euler-Poincaré characteristics (alternate sum of dimensions) is zero. We let the reader prove as a chasing exercise that the previous result is equivalent to prove that the following symbol sequence where $\operatorname{dim}\left(g_{r+2}\right)=r+4 \Rightarrow \operatorname{dim}\left(g_{r+4}\right)=r+6, \forall r \geq 0$ :

$$
0 \rightarrow g_{r+4} \rightarrow S_{r+4} T^{*} \otimes E \rightarrow S_{r+2} T^{*} \otimes F_{0} \rightarrow S_{r} T^{*} \otimes F_{1} \rightarrow 0
$$

is exact everywhere but at $S_{r+2} T^{*} \otimes F_{0}$ where the cohomology has dimension $r+2$, that is:

$$
\begin{aligned}
r+2 & =(2(r+3)(r+4) / 2-(r+1)(r+2) / 2)-((r+5)(r+6) / 2-(r+6)) \\
& =\operatorname{dim}\left(R_{r+3}\right)-\left(\operatorname{dim}\left(R_{r+4}\right)-\operatorname{dim}\left(g_{r+4}\right)\right)
\end{aligned}
$$

but such a method cannot be used for the more complicate examples dealing with GR that we shall find in the next section. Referring to the general theorem, we may consider the commutative diagram where $\mathcal{P}$ is an injective operator:

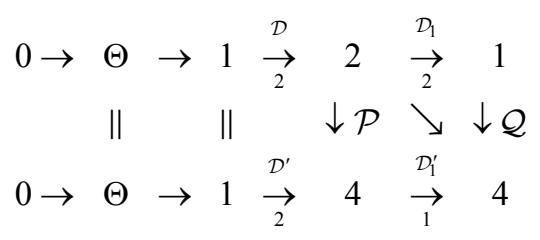

and the double prolongation diagram with $q=2, r=0, s=2, u \geq 0$ and $\mathcal{D}=\Phi \circ j_{q}, \mathcal{D}_{1}=\Psi_{1} \circ j_{2}$, where the two left upper downarrows are epimorphisms while the two left lower downarrows are monomorphisms:

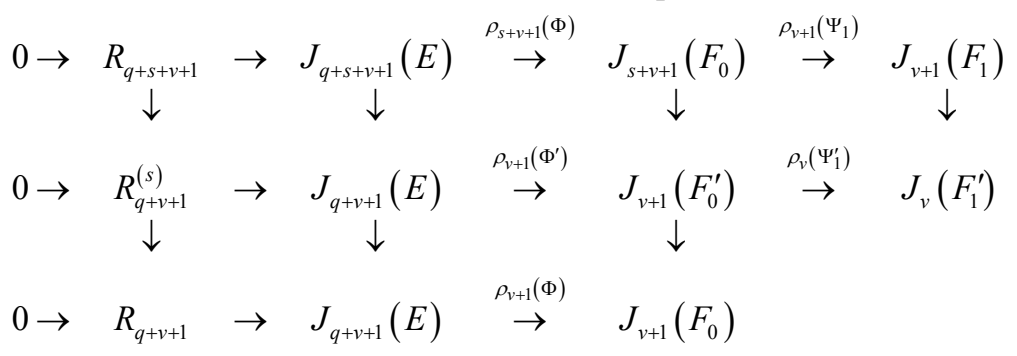

because we have been able to choose $\operatorname{ord}\left(\mathcal{D}_{1}\right)=r+s=s=2$ instead of $r+s+1$ as usual and where the columns are not sequences. Chasing now in an unusual way, we may start with any $b \in B_{s+u+1}=\operatorname{ker}\left(\rho_{u}\left(\Psi_{1}\right)\right)$ whenever $u \geq 0$ that we can lift to $\bar{b} \in B_{s+v+1}$ because we have chosen an involutive CC system, whenever $v \geq u \geq 0$. Choosing $v=r+s+u=s+u$, we may use the fact that the central line defines a Janet sequence and is thus an exact sequence. Therefore, choosing $\bar{c} \in J_{q+v+1}(E)$ such that $\rho_{v+1}\left(\Phi^{\prime}\right)(\bar{c})$ is the image of $\bar{b}$ in $J_{v+1}\left(F_{0}^{\prime}\right)$, we obtain by inclusion an element $c \in J_{q+v+1}(E)=J_{q+s+u+1}(E)$ such that $\rho_{v+1}(\Phi)(c)$ is $b$, ending the proof. In the differential module point of view, we have the commutative and exact diagram over $D=\mathbb{Q}\left[d_{1}, d_{2}, d_{3}\right]$ where the upper sequence comes from a Janet sequence:

$$
\begin{aligned}
& 0 \rightarrow D \underset{1}{\rightarrow} D^{4} \underset{1}{\rightarrow} D^{4} \underset{2}{\rightarrow} D \rightarrow M \rightarrow 0 \\
& \begin{array}{lllllllll} 
& & \downarrow & & \downarrow & & \| & & \| \\
0 & D & \rightarrow & D^{2} & \rightarrow & D & \rightarrow & M & \rightarrow 0
\end{array}
\end{aligned}
$$


The Euler-Poincaré characteristic, which is equal to the differential rank $r k_{D}(M)$ of $M$, vanishes in both resolutions which are called "exact" in algebraic analysis because infinite jets are implicitly used, even though the lower one is not "strictly" exact (Care, see [17] [18] [20] for more details).

\section{B) ALGEBROID BRACKET}

As we do not want to provide details about groupoids, we shall introduce a "copy" $Y$ (target) of $X$ (source) and define simply a Lie pseudogroup $\Gamma \subseteq \operatorname{aut}(X)$ as a group of transformations solutions of a (in general nonlinear) system $R_{q}$, such that, whenever $y=f(x), z=g(y) \in \Gamma$ can be composed, then $z=g \circ f(x) \in \Gamma, \quad x=f^{-1}(y) \in \Gamma \quad$ and $\quad y=i d(x)=x \in \Gamma$. Setting $y=x+t \xi(x)+\cdots$ and passing to the limit when $t \rightarrow 0$, we may linearize the later system and obtain a (linear) system $R_{q} \subset J_{q}(T)$ such that $[\Theta, \Theta] \subset \Theta$. We may use the Frobenius theorem in order to find a generating fundamental set of differential invariants $\left\{\Phi^{\tau}\left(y_{q}\right)\right\}$ up to order $q$ which are such that $\Phi^{\tau}\left(\bar{y}_{q}\right)=\Phi^{\tau}\left(y_{q}\right)$ whenever $\bar{y}=g(y) \in \Gamma$. We obtain the Lie form $\Phi^{\tau}\left(y_{q}\right)=\Phi_{\tau}\left(i d_{q}(x)\right)=\Phi^{\tau}\left(j_{q}(i d)(x)\right)=\omega^{\tau}(x)$ of $R_{q}$.

Of course, in actual practice one must use sections of $R_{q}$ instead of solutions and we now prove why the use of the Spencer operator becomes crucial for such a purpose. Indeed, using the algebraic bracket $\left\{j_{q+1}(\xi), j_{q+1}(\eta)\right\}=j_{q}([\xi, \eta]), \forall \xi, \eta \in T$, we may obtain by bilinearity a differential bracket on $J_{q}(T)$ extending the bracket on $T$ :

$$
\left[\xi_{q}, \eta_{q}\right]=\left\{\xi_{q+1}, \eta_{q+1}\right\}+i(\xi) D \eta_{q+1}-i(\eta) D \xi_{q+1}, \forall \xi_{q}, \eta_{q} \in J_{q}(T)
$$

which does not depend on the respective lifts $\xi_{q+1}$ and $\eta_{q+1}$ of $\xi_{q}$ and $\eta_{q}$ in $J_{q+1}(T)$. This bracket on sections satisfies the Jacobi identity ([12] [13] [14] [15] [17]):

$$
\left[\left[\xi_{q}, \eta_{q}\right], \zeta_{q}\right]+\left[\left[\eta_{q}, \zeta_{q}\right], \xi_{q}\right]+\left[\left[\zeta_{q}, \xi_{q}\right], \eta_{q}\right]=0
$$

and we set ([12] [13] [14] [15]):

DEFINITION 2B.1: We say that a vector subbundle $R_{q} \subset J_{q}(T)$ is a system of infinitesimal Lie equations or a Lie algebroid if $\left[R_{q}, R_{q}\right] \subset R_{q}$, that is to say $\left[\xi_{q}, \eta_{q}\right] \in R_{q}, \forall \xi_{q}, \eta_{q} \in R_{q}$. Such a definition can be tested by means of computer algebra. We shall also say that $R_{q}$ is transitive if we have the short exact sequence $0 \rightarrow R_{q}^{0} \rightarrow R_{q} \stackrel{\pi_{0}^{q}}{\longrightarrow} T \rightarrow 0$.

THEOREM 2B.2: The bracket is compatible with prolongations:

$$
\left[R_{q}, R_{q}\right] \subset R_{q} \Rightarrow\left[R_{q+r}, R_{q+r}\right] \subset R_{q+r}, \forall r \geq 0
$$

Proof: When $r=1$, we have

$\rho_{1}\left(R_{q}\right)=R_{q+1}=\left\{\xi_{q+1} \in J_{q+1}(T) \mid \xi_{q} \in R_{q}, D \xi_{q+1} \in T^{*} \otimes R_{q}\right\}$ and we just need to use the following formulas showing how $D$ acts on the various brackets if we set $L\left(\xi_{1}\right) \zeta=[\xi, \zeta]+i(\zeta) D \xi_{1}$ (see [12] and [15] for more details):

$$
\begin{aligned}
& i(\zeta) D\left\{\xi_{q+1}, \eta_{q+1}\right\}=\left\{i(\zeta) D \xi_{q+1}, \eta_{q}\right\}+\left\{\xi_{q}, i(\zeta) D \eta_{q+1}\right\}, \quad \forall \zeta \in T \\
& i(\zeta) D\left[\xi_{q+1}, \eta_{q+1}\right]= {\left[i(\zeta) D \xi_{q+1}, \eta_{q}\right]+\left[\xi_{q}, i(\zeta) D \eta_{q+1}\right] } \\
&+i\left(L\left(\eta_{1}\right) \zeta\right) D \xi_{q+1}-i\left(L\left(\xi_{1}\right) \zeta\right) D \eta_{q+1}
\end{aligned}
$$


The right member of the second formula is a section of $R_{q}$ whenever $\xi_{q+1}, \eta_{q+1} \in R_{q+1}$. The first formula may be used when $R_{q}$ is formally integrable.

Q.E.D.

COROLLARY 2B.3: The bracket is compatible with the PP procedure:

$$
\left[R_{q}, R_{q}\right] \subset R_{q} \Rightarrow\left[R_{q+r}^{(s)}, R_{q+r}^{(s)}\right] \subset R_{q+r}^{(s)}, \forall r, s \geq 0
$$

EXAMPLE 2B.4: With $n=1, q=3, X=\mathbb{R}$ and evident notations, the components of $\left[\xi_{3}, \eta_{3}\right]$ at order zero, one, two and three are defined by the totally unusual successive formulas:

$$
\begin{gathered}
{[\xi, \eta]=\xi \partial_{x} \eta-\eta \partial_{x} \xi} \\
\left(\left[\xi_{1}, \eta_{1}\right]\right)_{x}=\xi \partial_{x} \eta_{x}-\eta \partial_{x} \xi_{x} \\
\left(\left[\xi_{2}, \eta_{2}\right]\right)_{x x}=\xi_{x} \eta_{x x}-\eta_{x} \xi_{x x}+\xi \partial_{x} \eta_{x x}-\eta \partial_{x} \xi_{x x} \\
\left(\left[\xi_{3}, \eta_{3}\right]\right)_{x x x}=2 \xi_{x} \eta_{x x x}-2 \eta_{x} \xi_{x x x}+\xi \partial_{x} \eta_{x x x}-\eta \partial_{x} \xi_{x x x}
\end{gathered}
$$

Affine transformations: $\xi_{x x}=0, \eta_{x x}=0 \Rightarrow\left(\left[\xi_{2}, \eta_{2}\right]\right)_{x x}=0 \Rightarrow\left[R_{2}, R_{2}\right] \subset R_{2}$.

Projective transformations:

$\xi_{x x x}=0, \eta_{x x x}=0 \Rightarrow\left(\left[\xi_{3}, \eta_{3}\right]\right)_{x x x}=0 \Rightarrow\left[R_{3}, R_{3}\right] \subset R_{3}$.

EXAMPLE 2B.5: With $n=m=2$ and $q=1$, let us consider the Lie pseudodogroup $\Gamma \subset \operatorname{aut}(X)$ of finite transformations $y=f(x)$ such that $y^{2} d y^{1}=x^{2} d x^{1}=\alpha$. Setting $y=x+t \xi(x)+\cdots$ and linearizing, we get the Lie operator $\mathcal{D} \xi=\mathcal{L}(\xi) \alpha$ where $\mathcal{L}$ is the Lie derivative and the system $R_{1} \subset J_{1}(T)$ of linear infinitesimal Lie equations:

$$
x^{2} \partial_{1} \xi^{1}+\xi^{2}=0, \quad \partial_{2} \xi^{1}=0
$$

Replacing $j_{1}(\xi)$ by a section $\xi_{1} \in J_{1}(T)$, we have:

$$
\xi_{1}^{1}=-\frac{1}{x^{2}} \xi^{2}, \quad \xi_{2}^{1}=0
$$

Let us choose the two sections:

$$
\begin{gathered}
\xi_{1}=\left\{\xi^{1}=0, \xi^{2}=-x^{2}, \xi_{1}^{1}=1, \xi_{2}^{1}=0, \xi_{1}^{2}=0, \xi_{2}^{2}=0\right\} \in R_{1} \\
\eta_{1}=\left\{\eta^{1}=x^{2}, \eta^{2}=0, \eta_{1}^{1}=0, \eta_{2}^{1}=-x 2, \eta_{1}^{2}=0, \eta_{2}^{2}=1\right\} \in R_{1}
\end{gathered}
$$

We let the reader check that $\left[\xi_{1}, \eta_{1}\right] \in R_{1}$. However, we have the strict inclusion $R_{1}^{(1)} \subset R_{1}$ defined by the additional equation $\xi_{1}^{1}+\xi_{2}^{2}=0$ and thus $\xi_{1}, \eta_{1} \notin R_{1}^{(1)}$ though we have indeed $\left[R_{1}^{(1)}, R_{1}^{(1)}\right] \subset R_{1}^{(1)}$, a result not evident at all because $\xi_{1}$ and $\eta_{1}$ have nothing to do do with solutions. We invite the reader to proceed in the same way with $\beta=x^{2} d x^{1}-x^{1} d x^{2}$ and compare.

\section{C) EXTENSION MODULES}

Let $D=K\left[d_{1}, \cdots, d_{n}\right]=K[d]$ be the ring of differential operators with coefficients in a differential field $K$ of characteristic zero, that is such that $\mathbb{Q} \subset K$, with $n$ commuting derivations $\partial_{1}, \cdots, \partial_{n}$ and commutation relations 
$d_{i} a=a d_{i}+\partial_{i} a, \forall a \in K$. If $y^{1}, \cdots, y^{m}$ are $m$ differential indeterminates, we may identify $D y^{1}+\cdots+D y^{m}=D y$ with $D^{m}$ and consider the finitely presented left differential module $M={ }_{D} M$ with presentation $D^{p} \rightarrow D^{m} \rightarrow M \rightarrow 0$ determined by a given linear multidimensional system with $n$ independent variables, $m$ unknowns and $p$ equations. Applying the functor $\operatorname{hom}_{D}(\bullet, D)$, we get the exact sequence $0 \rightarrow \operatorname{hom}_{D}(M, D) \rightarrow D^{m} \rightarrow D^{p} \rightarrow N_{D} \rightarrow 0$ of right differential modules that can be transformed by a side-changing functor to an exact sequence of finitely generated left differential modules. This new presentation corresponds to the formal adjoint $a d(\mathcal{D})$ of the linear differential operator $\mathcal{D}$ determined by the initial presentation but now with $p$ unknowns and $m$ equations, obtaining therefore a new finitely generated left differential module $N={ }_{D} N$ and we may consider $\operatorname{hom}_{D}(M, D)$ as the module of equations of the compatibility conditions (CC) of ad $(\mathcal{D})$, a result not evident at first sight (see [16]). Using now a maximum free submodule $0 \rightarrow D^{l} \rightarrow \operatorname{hom}_{D}(M, D)$ and repeating this standard procedure while using the well known fact that $a d(\operatorname{ad}(\mathcal{D}))=\mathcal{D}$, we obtain therefore an embedding $0 \rightarrow \operatorname{hom}_{D}\left(\operatorname{hom}_{D}(M, D), D\right) \rightarrow D^{l}$ of left differential modules for a certain integer $1 \leq l<m$ because $K$ is a field and thus $D$ is a noetherian bimodule over itself, a result leading to $l=r k_{D}\left(\operatorname{hom}_{D}(M, D)\right)=r k_{D}(M)<m$ as in ([15] [16]). Now, setting $t(M)=\{m \in M \mid \exists 0 \neq P \in D, P m=0\}$, the kernel of the map $\epsilon: M \rightarrow \operatorname{hom}_{D}\left(\operatorname{hom}_{D}(M, D), D\right): m \rightarrow \epsilon(m)(f)=f(m), \forall f \in \operatorname{hom}_{D}(M, D) \quad$ is the torsion submodule $t(M) \subseteq M$ and $\epsilon$ is injective if and only if $M$ is torsion-free, that is $t(M)=0$. In that case, we obtain by composition an embedding $0 \rightarrow M \rightarrow D^{l}$ of $M$ into a free module. This result is quite important for applications as it provides a (minimal) parametrization of the linear differential operator $\mathcal{D}$ and amounts to the controllability of a classical control system when $n=1$ ([16] [34]). This parametrization will be called an "absolute parametrization" as it only involves arbitrary "potential-like" functions (see [16] [18] [20] [21] [24] [31] [33] [35] [36] [37] for more details and examples, in particular [34] for the Einstein equations).

If $P=a^{\mu} d_{\mu} \in D=K[d]$, the highest value of $|\mu|$ with $a^{\mu} \neq 0$ is called the order of the operator $P$ and the ring $D$ with multiplication $(P, Q) \rightarrow P \circ Q=P Q$ is filtred by the order $q$ of the operators. We have the filtration $0 \subset K=D_{0} \subset D_{1} \subset \cdots \subset D_{q} \subset \cdots \subset D_{\infty}=D$. Moreover, it is clear that $D$, as an algebra, is generated by $K=D_{0}$ and $T=D_{1} / D_{0}$ with $D_{1}=K \oplus T$ if we identify an element $\xi=\xi^{i} d_{i} \in T$ with the vector field $\xi=\xi^{i}(x) \partial_{i}$ of differential geometry, but with $\xi^{i} \in K$ now. It follows that $D={ }_{D} D_{D}$ is a bimodule over itself, being at the same time a left $D$-module by the composition $P \rightarrow Q P$ and a right $D$-module by the composition $P \rightarrow P Q$. We define the adjoint map ad $: D \rightarrow D^{o p}: P=a^{\mu} d_{\mu} \rightarrow a d(P)=(-1)^{|\mu|} d_{\mu} a^{\mu}$ and we have $\operatorname{ad}(\operatorname{ad}(P))=P$. It is easy to check that $\operatorname{ad}(P Q)=a d(Q) a d(P), \forall P, Q \in D$. Such a definition can also be extended to any matrix of operators by using the transposed matrix of adjoint operators (see [21] [23] [25] [34] for more details 
or applications to control theory and mathematical physics).

Accordingly, if $y=\left(y^{1}, \cdots, y^{m}\right)$ are differential indeterminates, then $D$ acts on $y^{k}$ by setting $d_{\mu} y^{k}=y_{\mu}^{k}$ with $d_{i} y_{\mu}^{k}=y_{\mu+1_{i}}^{k}$ and $y_{0}^{k}=y^{k}$. We may therefore use the jet coordinates in a formal way as in the previous section. Therefore, if a system of OD/PD equations is written in the form:

$$
\Phi^{\tau} \equiv a_{k}^{\tau \mu} y_{\mu}^{k}=0
$$

with coefficients $a_{k}^{\tau \mu} \in K$, we may introduce the free differential module $D y=D y^{1}+\cdots+D y^{m} \simeq D^{m}$ and consider the differential submodule $I=D \Phi \subset D y$ which is usually called the module of equations, both with the differential module $M=D y / D \Phi$ or $D$-module and we may set $M={ }_{D} M$ if we want to specify the ring of differential operators. Again, we may introduce the formal prolongation with respect to $d_{i}$ by setting:

$$
d_{i} \Phi^{\tau} \equiv a_{k}^{\tau \mu} y_{\mu+1_{i}}^{k}+\left(\partial_{i} a_{k}^{\tau \mu}\right) y_{\mu}^{k}
$$

with $\mu+1_{i}=\left(\mu_{1}, \cdots, \mu_{i-1}, \mu_{i}+1, \mu_{i+1}, \cdots, \mu_{n}\right)$ in order to induce maps $d_{i}: M \rightarrow M: \bar{y}_{\mu}^{k} \rightarrow \bar{y}_{\mu+1_{i}}^{k}$ by residue if we use to denote the residue $D y \rightarrow M: y^{k} \rightarrow \bar{y}^{k}$ by a bar as in algebraic geometry. However, for simplicity, we shall not write down the bar when the background will indicate clearly if we are in $D y$ or in $M$. We have a filtration

$0 \subseteq M_{0} \subseteq M_{1} \subseteq \cdots \subseteq M_{q} \subseteq \cdots \subseteq M_{\infty}=M$ induced by that of $D$ and $d_{i} M_{q} \subseteq M_{q+1}$ (compare to [35] and [37]).

As a byproduct, the differential modules we shall consider will always be finitely generated $(k=1, \cdots, m<\infty)$ and finitely presented $(\tau=1, \cdots, p<\infty)$. Equivalently, introducing the matrix of operators $\mathcal{D}=\left(a_{k}^{\tau \mu} d_{\mu}\right)$ with $m$ columns and $p$ rows, we may introduce the morphism

$D^{p} \stackrel{\mathcal{D}}{\longrightarrow} D^{m}:\left(P_{\tau}\right) \rightarrow\left(P_{\tau} \Phi^{\tau}\right): P \rightarrow P \Phi=P \mathcal{D}$ over $D$ by acting with $\mathcal{D}$ on the left of these row vectors while acting with $D$ on the right of these row vectors and the presentation of $M$ is defined by the exact cokernel sequence $D^{p} \rightarrow D^{m} \rightarrow M \rightarrow 0$. It is essential to notice that the presentation only depends on $K, D$ and $\Phi$ or $\mathcal{D}$, that is to say never refers to the concept of (explicit or formal) solutions. It is at this moment that we have to take into account the results of the previous section in order to understant that certain presentations will be much better than others, in particular to establish a link with formal integrability and involution.

Having in mind that $K$ is a left $D$-module with the standard action $(D, K) \rightarrow K:\left(d_{i}, a\right) \rightarrow \partial_{i} a$ and that $D$ is a bimodule over itself, we have only two possible constructions:

DEFINITION 2C.1: We define the system $R=\operatorname{hom}_{K}(M, K)=M^{*}$ and set $R_{q}=\operatorname{hom}_{K}\left(M_{q}, K\right)=M_{q}^{*}$ as the system of order $q$. We have the projective limit $R=R_{\infty} \rightarrow \cdots \rightarrow R_{q} \rightarrow \cdots \rightarrow R_{1} \rightarrow R_{0}$. It follows that $f_{q} \in R_{q}: y_{\mu}^{k} \rightarrow f_{\mu}^{k} \in K$ with $a_{k}^{\tau \mu} f_{\mu}^{k}=0$ defines a section at order $q$ and we may set $f_{\infty}=f \in R$ for a section of $R$. For a ground field of constants $k$, this definition has of course to do with the concept of a formal power series solution. 
However, for an arbitrary differential field $K$, the main novelty of this new approach is that such a definition has nothing to do with the concept of a formal power series solution (care) as illustrated in the next example.

DEFINITION 2C.2: We may define the right differential module $\operatorname{hom}_{D}(M, D)$.

PROPOSITION 2C.3: When $M$ is a left $D$-module, then $R$ is also a left $D$-module.

Proof. As $D$ is generated by $K$ and $T$ as we already said, let us define:

$$
\begin{gathered}
(a f)(m)=a f(m), \quad \forall a \in K, \forall m \in M \\
(\xi f)(m)=\xi f(m)-f(\xi m), \quad \forall \xi=a^{i} d_{i} \in T, \forall m \in M
\end{gathered}
$$

In the operator sense, it is easy to check that $d_{i} a=a d_{i}+\partial_{i} a$ and that $\xi \eta-\eta \xi=[\xi, \eta]$ is the standard bracket of vector fields. We finally get $\left(d_{i} f\right)_{\mu}^{k}=\left(d_{i} f\right)\left(y_{\mu}^{k}\right)=\partial_{i} f_{\mu}^{k}-f_{\mu+1_{i}}^{k}$ and thus recover exactly the Spencer operator of the previous section though this is not evident at all. We also get $\left(d_{i} d_{j} f\right)_{\mu}^{k}=\partial_{i j} f_{\mu}^{k}-\partial_{i} f_{\mu+1_{j}}^{k}-\partial_{j} f_{\mu+1_{i}}^{k}+f_{\mu+1_{i}+1_{j}}^{k} \Rightarrow d_{i} d_{j}=d_{j} d_{i}, \forall i, j=1, \cdots, n \quad$ and thus $\quad d_{i} R_{q+1} \subseteq R_{q} \Rightarrow d_{i} R \subset R \quad$ induces a well defined operator $R \rightarrow T^{*} \otimes R: f \rightarrow d x^{i} \otimes d_{i} f$. This result has been discovered (up to sign) by Macaulay in 1916 ([33]). For more details on the Spencer operator and its applications, the reader may look at ([14] [15]).

Q.E.D.

We now recall the definition of the extension modules $\operatorname{ext}_{D}^{i}(M, D)$ that we shall simply denote by $\operatorname{ext}^{i}(M)$ when there cannot be any confusion. We divide the procedure into four steps that can be achieved by means of computer algebra ([17] [36] [38] [39]):

- Construct a free resolution of $M$, say:

$$
\cdots \rightarrow F_{i} \rightarrow \cdots \rightarrow F_{1} \rightarrow F_{0} \rightarrow M \rightarrow 0
$$

- Suppress $M$ in order to obtain the deleted sequence:

$$
\cdots \rightarrow F_{i} \rightarrow \cdots \rightarrow F_{1} \rightarrow F_{0} \rightarrow 0
$$

- Apply $\operatorname{hom}_{D}(\bullet, D)$ in order to obtain the dual sequence heading backwards:

$$
\cdots \leftarrow \operatorname{hom}_{D}\left(F_{i}, D\right) \leftarrow \cdots \leftarrow \operatorname{hom}_{D}\left(F_{1}, D\right) \leftarrow \operatorname{hom}_{D}\left(F_{0}, D\right) \leftarrow 0
$$

- Define $\operatorname{ext}^{i}(M)$ to be the cohomology at $\operatorname{hom}_{D}\left(F_{i}, D\right)$ in the dual sequence with $\operatorname{ext}^{0}(M)=\operatorname{hom}_{D}(M, D)$.

The following nested chain of difficult propositions and theorems can be obtained, even in the non-commutative case, by combining the use of extension modules and double duality in the framework of algebraic analysis ([16] [35] [39]).

THEOREM 2C.4: The extension modules do not depend on the resolution of $M$ used.

PROPOSITION 2C.5: Applying $\operatorname{hom}_{D}(\bullet, D)$ provides right $D$-modules that can be transformed to left $D$-modules by means of the side changing functor and 
vice-versa. Namely, if $N_{D}$ is a right $D$-module, then ${ }_{D} N=\wedge^{n} T \otimes_{K} N_{D}$ is the converted left $D$-module while, if ${ }_{D} N$ is a left $D$-module, then $N_{D}=\wedge^{n} T^{*} \otimes_{K D} N$ is the converted right $D$-module.

PROPOSITION 2C.6: Instead of using $\operatorname{hom}_{D}(\bullet, D)$ and the side changing functor in the module framework, we may use $a d$ in the operator framework. Namely, to any operator $\mathcal{D}: E \rightarrow F$ we may associate the formal adjoint $\operatorname{ad}(\mathcal{D}): \wedge^{n} T^{*} \otimes F^{*} \rightarrow \wedge^{n} T^{*} \otimes E^{*}$ with the useful though striking relation $r k_{D}(\operatorname{ad}(\mathcal{D}))=r k_{D}(\mathcal{D})$.

PROPOSITION 2.C.7: $\operatorname{ext}^{i}(M)$ is a torsion module $\forall 1 \leq i \leq n$ but $\operatorname{ext}^{0}(M)=\operatorname{hom}_{D}(M, D)$ may not be a torsion module.

We shall say that an operator is parametrizable if it generates the CC of an operator and the next result will be essential for applications as it can be tested by means of computer algebra ([38]).

THEOREM 2C.8: An operator is parametrizable if and only if the corresponding differential module is torsion-free and double duality provides a constructive test for checking such a property.

\section{Applications}

\section{A) Minkowski Metric:}

If $n=4$ and $\omega \in S_{2} T^{*}$ is the non-degenerate Minkowski metric, the corresponding Lie operator is $\xi \in T \rightarrow \Omega \equiv \mathcal{D} \xi=\mathcal{L}(\xi) \omega \in S_{2} T^{*}$ where $\mathcal{L}$ is the standard Lie derivative for tensors and we have to study the corresponding system $R_{1} \subset J_{1}(T)$ of infinitesimal Lie equations. However, this system is finite type with $g_{2}=0$ but $g_{1} \subset T^{*} \otimes T$ is not 2 -acyclic and the CC are homogeneous of order 2, a result leading to the well-known finite length differential sequence where the order of an operator has been indicated under its arrow:

$$
0 \rightarrow \Theta \rightarrow 4 \underset{1}{\stackrel{\text { Killing }}{\rightarrow}} 10 \underset{2}{\stackrel{\text { Riemann }}{\rightarrow}} 20 \underset{1}{\stackrel{\text { Bianchi }}{\rightarrow}} 20 \underset{1}{\rightarrow} 6 \rightarrow 0
$$

In arbitrary dimension, we have successively:

$$
n \rightarrow n(n+1) / 2 \rightarrow n^{2}\left(n^{2}-1\right) / 12 \rightarrow n^{2}\left(n^{2}-1\right)(n-2) / 24 \rightarrow \cdots
$$

or, introducing the Spencer $\delta$-cohomology:

$$
T \rightarrow S_{2} T^{*} \rightarrow H^{2}\left(g_{1}\right) \rightarrow H^{3}\left(g_{1}\right) \rightarrow \cdots
$$

However, this sequence is not canonical and we have to use the involutive system $R_{2} \subset J_{2}(T)$ in the following Fundamental Diagram $I$ relating the (upper) canonical Spencer sequence to the (lower) canonical Janet sequence, a result first found exactly 40 years ago in ([12]) that only depends on the left commutative square $\mathcal{D}=\Phi_{0} \circ j_{q}$ when one has an involutive system $R_{q} \subseteq J_{q}(E)$ over $E$ and thus the involutive system $R_{q+1} \subset J_{1}\left(R_{q}\right)$ over $R_{q}$ when $\operatorname{dim}(X)=n$. 


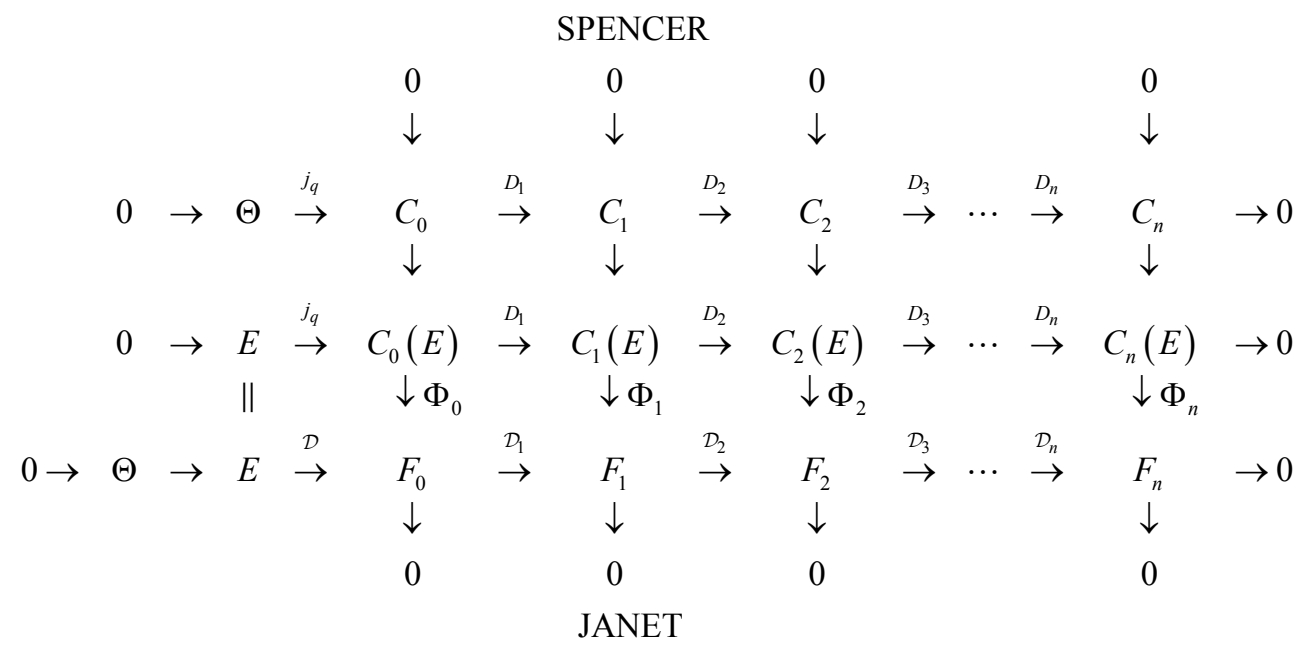

Setting $n=4, q=2, E=T, C_{r}=\wedge^{r} T^{*} \otimes R_{2}$, the first order first Spencer operator $D_{1}$ is defined by the system $R_{3} \subset J_{1}\left(R_{2}\right)$ and the epimorphisms $\Phi_{1}, \cdots, \Phi_{4}$ are induced by the epimorphism $\Phi_{0}$. The corresponding fiber dimensions are indicated in the next diagram where

$$
\begin{aligned}
& \operatorname{dim}\left(R_{2}\right)=\operatorname{dim}(T)+\operatorname{dim}\left(g_{1}\right)=4+6=10 \text { : } \\
& \begin{array}{lllll}
0 & 0 & 0 & 0 & 0 \\
\downarrow & \downarrow & \downarrow & \downarrow & \downarrow
\end{array}
\end{aligned}
$$

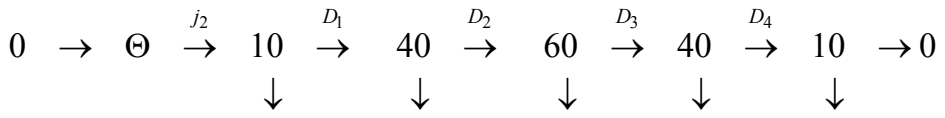

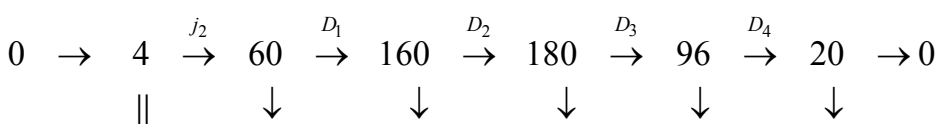

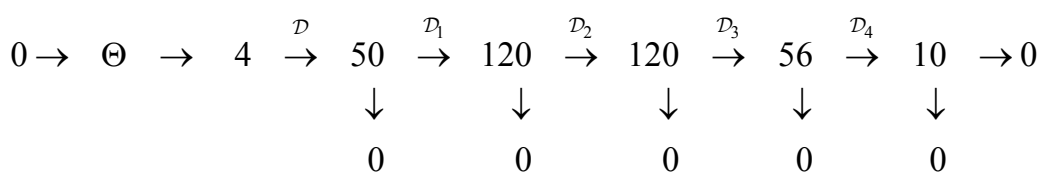

We recall that, apart from $j_{2}$ and $\mathcal{D}=\Phi_{0} \circ j_{2}$ which have order 2, then all the other operators have order 1 . Of course, the reader may imagine easily that the author of this paper avoided computer algebra by using the following specific procedure:

1) First write down the Spencer sequence at once, keeping in mind that it is locally isomorphic to the tensor product of the Poincaré sequence:

$$
\begin{gathered}
\wedge^{0} T^{*} \stackrel{d}{\longrightarrow} \wedge^{1} T^{*} \stackrel{d}{\longrightarrow} \wedge^{2} T^{*} \stackrel{d}{\longrightarrow} \wedge^{3} T^{*} \stackrel{d}{\longrightarrow} \wedge^{4} T^{*} \rightarrow 0 \\
1 \stackrel{d}{\longrightarrow} 4 \stackrel{d}{\longrightarrow} 6 \stackrel{d}{\longrightarrow} 4 \stackrel{d}{\longrightarrow} 1 \rightarrow 0
\end{gathered}
$$

by a Lie algebra of dimension $4+6=10$ and is thus locally exact.

2) Second, write down the central sequence just using some combinatorics on the Janet tabular for the trivially involutive second order operator $j_{2}$ (see [12], p. 157).

3) Then, obtain the Janet sequence by quotient.

4) Finally, use the vanishing of the three Euler-Poincare characteristics for 
checking the exactness of all these numbers, namely:

$$
\begin{aligned}
& 10-40+60-40+4=0, \\
& 4-60+160-180+96-20=0, \\
& 4-50+120-120+56-10=0
\end{aligned}
$$

\section{B) SCHWARZSCHILD METRIC:}

In the Boyer-Lindquist coordinates $(t, r, \theta, \phi)=\left(x^{0}, x^{1}, x^{2}, x^{3}\right)$, we may consider the Schwarzschild metric

$\omega=A(r) d t^{2}-(1 / A(r)) d r^{2}-r^{2} d \theta^{2}-r^{2} \sin ^{2}(\theta) d \phi^{2}$ and $\xi=\xi^{i} d_{i} \in T$, let us introduce $\xi_{i}=\omega_{r i} \xi^{r}$ with the 4 formal derivatives $\left(d_{0}=d_{t}, d_{1}=d_{r}, d_{2}=d_{\theta}, d_{3}=d_{\phi}\right)$. With speed of light $c=1$ and $A=1-\frac{m}{r}$ where $m$ is a constant, the metric can be written in the diagonal form:

$$
\left(\begin{array}{cccc}
A & 0 & 0 & 0 \\
0 & -1 / A & 0 & 0 \\
0 & 0 & -r^{2} & 0 \\
0 & 0 & 0 & -r^{2} \sin ^{2}(\theta)
\end{array}\right)
$$

Using the notations of differential modules theory, consider the Killing equations:

$$
\Omega \equiv L(\xi) \omega=0 \Leftrightarrow \Omega_{i j} \equiv d_{i} \xi_{j}+d_{j} \xi_{i}-2 \gamma_{i j}^{r} \xi_{r}=0
$$

where we have introduced the Christoffel symbols $\gamma$ through he standard Levi-Civita isomorphism $j_{1}(\omega) \simeq(\omega, \gamma)$ while setting $A^{\prime}=\partial_{r} A$ in the differential field $K$ of coefficients ([40], p. 87). As in the later Macaulay and Janet examples and in order to avoid any further confusion between sections and derivatives, we shall use the sectional point of view and rewrite the previous equations in the symbolic form $\Omega \equiv L\left(\xi_{1}\right) \omega \in S_{2} T^{*}$ where $L$ is the formal Lie derivative:

$$
\left\{\begin{array}{l}
\frac{1}{2} \Omega_{33} \equiv \xi_{3,3}+\sin (\theta) \cos (\theta) \xi_{2}+r A \sin ^{2}(\theta) \xi_{1}=0 \\
\Omega_{32} \equiv \xi_{2,3}+\xi_{3,2}-2 \cot (\theta) \xi_{3}=0 \\
\Omega_{31} \equiv \xi_{1,3}+\xi_{3,1}-\frac{2}{r} \xi_{3}=0 \\
\Omega_{30} \equiv \xi_{0,3}+\xi_{3,0}=0 \\
\frac{1}{2} \Omega_{22} \equiv \xi_{2,2}+r A \xi_{1}=0 \\
\Omega_{21} \equiv \xi_{1,2}+\xi_{2,1}-\frac{2}{r} \xi_{2}=0 \\
\Omega_{20} \equiv \xi_{0,2}+\xi_{2,0}=0 \\
\frac{1}{2} \Omega_{11} \equiv \xi_{1,1}+\frac{A^{\prime}}{2 A} \xi_{1}=0 \\
\Omega_{10} \equiv \xi_{0,1}+\xi_{1,0}-\frac{A^{\prime}}{A} \xi_{0}=0 \\
\frac{1}{2} \Omega_{00} \equiv \xi_{0,0}-\frac{A A^{\prime}}{2} \xi_{1}=0
\end{array}\right.
$$


Though this system $R_{1} \subset J_{1}(T)$ has 4 equations of class 3,3 equations of class 2, 2 equations of class 1 and 1 equation of class 0 , it is far from being involutive because it is finite type with second symbol $g_{2}=0$ defined by the 40 equations $v_{i j}^{k}=0$ in the initial coordinates. From the symmetry, it is clear that such a system has at least 4 solutions, namely the time translation $\partial_{t} \leftrightarrow \xi^{0}=1 \Leftrightarrow \xi_{0}=A$ and, using cartesian coordinates $(t, x, y, z)$, the 3 space rotations $y \partial_{z}-z \partial_{y}, z \partial_{x}-x \partial_{z}, x \partial_{y}-y \partial_{x}$.

We may also write the Schwarzschild metric in cartesian coordinates as:

$$
\omega=A(r) d t^{2}+\left(1-\frac{1}{A(r)}\right) d r^{2}-\left(d x^{2}+d y^{2}+d z^{2}\right), \quad r d r=x d x+y d y+z d z
$$

and notice that the $3 \times 3$ matrix of components of the three rotations has rank equal to 2, a result leading surely, before doing any computation to the existence of one and only one zero order Killing equation $x \xi^{x}+y \xi^{y}+z \xi^{z}=0$. Such a result also amounts to say that the spatial projection of any Killing vector on the radial spatial unit vector $\left(x^{1} / r, x^{2} / r, x^{3} / r\right)$ vanishes because $r$ must stay invariant.

Caring only about the last three equations, we get formally:

$$
\begin{aligned}
& \xi_{0,11}+\xi_{1,01}-d_{1}\left(\frac{A^{\prime}}{A} \xi_{0}\right) \\
& =\xi_{0,11}-\frac{A^{\prime}}{2 A} \xi_{1,0}-\left(\frac{A^{\prime \prime}}{A}-\frac{\left(A^{\prime}\right)^{2}}{A^{2}}\right) \xi_{0}-\frac{A^{\prime}}{A} \xi_{0,1} \\
& =\xi_{0,11}+\frac{A^{\prime}}{2 A} \xi_{0,1}-\frac{\left(A^{\prime}\right)^{2}}{2 A^{2}} \xi_{0}-\left(\frac{A^{\prime \prime}}{A}-\frac{\left(A^{\prime}\right)^{2}}{A^{2}}\right) \xi_{0}-\frac{A^{\prime}}{A} \xi_{0,1} \\
& =\xi_{0,11}+\frac{A^{\prime}}{2 A} \xi_{1,0}-\frac{A^{\prime \prime}}{A} \xi_{0}
\end{aligned}
$$

We obtain the linearized Christoffel symbols $\Gamma=L\left(\xi_{2}\right) \gamma \in S_{2} T^{*} \otimes T$ in the form:

$$
\Gamma_{0,11} \equiv \xi_{0,11}+\frac{A^{\prime}}{2 A} \xi_{1,0}-\frac{A^{\prime \prime}}{A} \xi_{0}=0
$$

and similarly in $R_{2}$ with lower indices as usual:

$$
\begin{gathered}
\Gamma_{0,01} \equiv \xi_{0,01}-\left(\frac{A A^{\prime \prime}}{2}+\frac{\left(A^{\prime}\right)^{2}}{4}\right) \xi_{1}=0 \\
\Gamma_{0,00} \equiv \xi_{0,00}-\frac{A A^{\prime}}{2} \xi_{1,0}=0 \\
\Gamma_{1,00} \equiv \xi_{1,00}+\left(\frac{A A^{\prime \prime}}{2}-\frac{A^{\prime 2}}{4}\right) \xi_{1}=0 \\
\Gamma_{1,01} \equiv \xi_{1,01}+\frac{A^{\prime}}{2 A} \xi_{1,0}=0 \\
\Gamma_{1,11} \equiv \xi_{1,11}+\left(\frac{A^{\prime \prime}}{2 A}-\frac{3\left(A^{\prime}\right)^{2}}{4 A^{2}}\right) \xi_{1}=0
\end{gathered}
$$


It follows that we obtain in $R_{3}$ and thus finally in $R_{1}^{(2)}$ for constructing $\rho_{01,01}$ :

$$
\begin{aligned}
& +\xi_{1,001}+\frac{A^{\prime}}{2 A} \xi_{1,00}=0 \\
& -\xi_{1,001}-\left(\frac{A A^{\prime \prime}}{2}-\frac{\left(A^{\prime}\right)^{2}}{4}\right)^{\prime} \xi_{1}-\left(\frac{A A^{\prime \prime}}{2}-\frac{\left(A^{\prime}\right)^{2}}{4}\right) \xi_{1,1}=0 \\
& -\frac{A^{\prime}}{2 A} \xi_{1,00}-\frac{A^{\prime}}{2 A}\left(\frac{A A^{\prime \prime}}{2}-\frac{\left(A^{\prime}\right)^{2}}{4}\right) \xi_{1}=0 \\
& \left(\frac{A A^{\prime \prime}}{2}-\frac{\left(A^{\prime}\right)^{2}}{4}\right) \xi_{1,1}+\frac{A^{\prime}}{2 A}\left(\frac{A A^{\prime \prime}}{2}-\frac{\left(A^{\prime}\right)^{2}}{4}\right) \xi_{1}=0
\end{aligned}
$$

Summing these four prolongations, we get $\xi^{1} d_{1}\left(\frac{A A^{\prime \prime}}{2}-\frac{\left(A^{\prime}\right)^{2}}{4}\right)=0 \Rightarrow \xi_{1}=0$ because $A=1-\frac{m}{r} \Rightarrow A+r A^{\prime}=1 \Rightarrow 2 A^{\prime}+r A^{\prime \prime}=0$. Similarly, we could have obtained:

$$
d_{1} \xi_{1,00}-d_{0} \xi_{1,01}=-\left(\frac{A A^{\prime \prime}}{2}-\frac{\left(A^{\prime}\right)^{2}}{4}\right) \xi_{1}=0
$$

Using the relation $A+r A^{\prime}=1$, we have also successively for constructing $\rho_{02,01}$ :

$$
\begin{gathered}
d_{1} \Omega_{02}+d_{0} \Omega_{12}-d_{2} \Omega_{01}=2 \xi_{2,01}-\left(\frac{2}{r}+\frac{A^{\prime}}{A}\right) \xi_{2,0} \Rightarrow \xi_{2,01}=\left(\frac{1}{r}+\frac{A^{\prime}}{2 A}\right) \xi_{2,0} \\
\xi_{2,00}=-\xi_{0,20}=-\frac{A A^{\prime}}{2} \xi_{1,2}, \quad \xi_{0,12}=-\left(\frac{1}{r}+\frac{A^{\prime}}{2 A}\right) \xi_{2,0} \\
d_{1}\left(\xi_{2,00}\right)-d_{0}\left(\xi_{2,01}\right)-\frac{\left(A A^{\prime}\right)^{\prime}}{2} \xi_{1,2}-\frac{A A^{\prime}}{2} \xi_{1,12}-\left(\frac{1}{r}+\frac{A^{\prime}}{2 A}\right) \xi_{2,00} \\
=-\left(\frac{A A^{\prime \prime}}{2}+\frac{\left(A^{\prime}\right)^{2}}{4}\right) \xi_{1,2}+\frac{A A^{\prime}}{2}\left(\frac{1}{r}+\frac{A^{\prime}}{2 A}\right) \xi_{1,2} \\
=\left(\frac{A A^{\prime}}{2 r}-\frac{A A^{\prime \prime}}{2}\right) \xi_{1,2}=\frac{3 A A^{\prime}}{2 r} \xi_{1,2}
\end{gathered}
$$

that is to say $\xi_{1,2}=0$. However, we have:

$$
\begin{aligned}
d_{1}\left(\xi_{1,01}\right)-d_{0}\left(\xi_{1,11}\right) & =d_{1}\left(-\frac{A^{\prime}}{2 A} \xi_{1,0}\right)-\left(\frac{3\left(A^{\prime}\right)^{2}}{4 A^{2}}-\frac{A^{\prime \prime}}{2 A}\right) \xi_{1,0} \\
& =-\frac{A^{\prime}}{2 A} \xi_{1,01}-\left(\frac{A^{\prime \prime}}{2 A}-\frac{\left(A^{\prime}\right)^{2}}{2 A^{2}}\right) \xi_{1,0}-\left(\frac{3\left(A^{\prime}\right)^{2}}{4 A^{2}}-\frac{A^{\prime \prime}}{2 A}\right) \xi_{1,0} \\
& =\left(\frac{\left(A^{\prime}\right)^{2}}{4 A^{2}}+\frac{\left(A^{\prime}\right)^{2}}{2 A^{2}}-\frac{3\left(A^{\prime}\right)^{2}}{4 A^{2}}\right) \xi_{1,0}=0
\end{aligned}
$$


and such an approach does not bring $\xi_{1,0}=0$ for sections, even if it must be surely true for solutions. We may also notice that:

$$
\begin{gathered}
d_{1} \Omega_{02}+d_{2} \Omega_{01}-d_{0} \Omega_{12}=2 \xi_{0,12}-\frac{A^{\prime}}{A} \xi_{0,2}+\frac{2}{r} \xi_{2,0} \\
\Rightarrow \xi_{0,12}=\left(\frac{A^{\prime}}{2 A}+\frac{1}{r}\right) \xi_{0,2} \\
d_{0} \Omega_{12}+d_{2} \Omega_{01}-d_{1} \Omega_{02}=2 \xi_{1,02}-\frac{2}{r} \xi_{2,0}-\frac{A^{\prime}}{A} \xi_{0,2} \\
\Rightarrow \xi_{1,02}=\left(\frac{A^{\prime}}{2 A}-\frac{1}{r}\right) \xi_{0,2} \\
\xi_{0,11}=\frac{A^{\prime \prime}}{A} \xi_{0}-\frac{A^{\prime}}{2 A} \xi_{1,0}
\end{gathered}
$$

Studying the component $\rho_{01,12}$, we obtain successively:

$$
\begin{aligned}
d_{2} \xi_{0,11}-d_{1} \xi_{0,12} & =\frac{A^{\prime \prime}}{A} \xi_{0,2}-\frac{A^{\prime}}{2 A} \xi_{1,02}-\left(\frac{A^{\prime}}{2 A}+\frac{1}{r}\right)^{\prime} \xi_{0,2}-\left(\frac{A^{\prime}}{2 A}+\frac{1}{r}\right) \xi_{0,12} \\
& =\left[\frac{A^{\prime \prime}}{A}-\frac{A^{\prime}}{2 A}\left(\frac{A^{\prime}}{2 A}-\frac{1}{r}\right)-\left(\frac{A^{\prime}}{2 A}+\frac{1}{r}\right)^{\prime}-\left(\frac{A^{\prime}}{2 A}+\frac{1}{r}\right)^{2}\right] \xi_{0,2} \\
& =-\frac{2 A^{\prime}}{r A} \xi_{0,2}
\end{aligned}
$$

and thus $\xi_{0,2}=0$. We also have:

$$
\begin{gathered}
d_{3} \Omega_{01}+d_{1} \Omega_{03}-d_{0} \Omega_{13}=2 \xi_{0,13}-\frac{A^{\prime}}{A} \xi_{0,3}+\frac{2}{r} \xi_{3,0} \\
\Rightarrow \xi_{0,13}=\left(\frac{A^{\prime}}{2 A}+\frac{1}{r}\right) \xi_{0,3} \\
d_{0} \Omega_{13}+d_{3} \Omega_{01}-d_{1} \Omega_{03}=2 \xi_{1,03}-\frac{2}{r} \xi_{3,0}-\frac{A^{\prime}}{A} \xi_{0,3} \\
\Rightarrow \xi_{1,03}=\left(\frac{A^{\prime}}{2 A}-\frac{1}{r}\right) \xi_{0,3}
\end{gathered}
$$

Studying the component $\rho_{01,13}$, we obtain successively:

$$
\begin{gathered}
\xi_{0,11}=\frac{A^{\prime \prime}}{A} \xi_{0}-\frac{A^{\prime}}{2 A} \xi_{1,0} \\
d_{3} \Omega_{01}+d_{1} \Omega_{03}-d_{0} \Omega_{13}=2 \xi_{0,13}-\frac{A^{\prime}}{A} \xi_{0,3} \\
d_{0} \Omega_{13}+d_{3} \Omega_{01}-d_{1} \Omega_{0,3}=2 \xi_{1,03}-\frac{2}{r} \xi_{3,0}-\frac{A^{\prime}}{A} \xi_{0,3} \\
\Rightarrow \xi_{1,03}=\left(\frac{A^{\prime}}{2 A}-\frac{1}{r}\right) \xi_{0,3}
\end{gathered}
$$




$$
\begin{aligned}
d_{3} \xi_{0,11}-d_{1} \xi_{0,13} & =\frac{A^{\prime \prime}}{A} \xi_{0,3}-\frac{A^{\prime}}{2 A} \xi_{1,03}-\left(\frac{A^{\prime}}{2 A}+\frac{1}{r}\right)^{\prime} \xi_{0,3}-\left(\frac{A^{\prime}}{2 A}+\frac{1}{r}\right) \xi_{0,13} \\
& =\left[\frac{A^{\prime \prime}}{A}-\frac{A^{\prime}}{2 A}\left(\frac{A^{\prime}}{2 A}-\frac{1}{r}\right)-\left(\frac{A^{\prime}}{2 A}+\frac{1}{r}\right)^{\prime}-\left(\frac{A^{\prime}}{2 A}+\frac{1}{r}\right)^{2}\right] \xi_{0,3} \\
& =-\frac{3 A^{\prime}}{2 r A} \xi_{0,3}
\end{aligned}
$$

and thus $\xi_{0,3}=0$. We also have:

$$
\begin{aligned}
& d_{1} \Omega_{03}+d_{0} \Omega_{13}-d_{3} \Omega_{01}=2 \xi_{3,01}-\frac{2}{r} \xi_{3,0}-\frac{A^{\prime}}{A} \xi_{0,3} \\
\Rightarrow & \xi_{3,01}=\left(\frac{A^{\prime}}{2 A}-\frac{1}{r}\right) \xi_{0,3}, \quad \xi_{3,00}=-\xi_{0,03}=-\frac{A A^{\prime}}{2} \xi_{1,3}
\end{aligned}
$$

Studying $\rho_{03,01}$, we obtain successively:

$$
\begin{aligned}
d_{0} \xi_{3,01}-d_{1} \xi_{3,00} & =\left(\frac{A^{\prime}}{2 A}-\frac{1}{r}\right) \xi_{0,03}+\left(\frac{A A^{\prime}}{2}\right)^{\prime} \xi_{1,3}+\frac{A A^{\prime}}{2} \xi_{1,13} \\
& =\left[\frac{A A^{\prime}}{2}\left(\frac{A^{\prime}}{2 A}-\frac{1}{r}\right)+\left(\frac{A A^{\prime}}{2}\right)^{\prime}-\frac{\left(A^{\prime}\right)^{2}}{4}\right] \xi_{1,3} \\
& =\left(\frac{A A^{\prime}}{2 r}+\left(\frac{A A^{\prime}}{2}\right)^{\prime}\right) \xi_{1,3}
\end{aligned}
$$

and thus $\xi_{1,3}=0$. We also have:

$$
\begin{aligned}
d_{0} \Omega_{12}+d_{1} \Omega_{02} & -d_{2} \Omega_{01}=2 \xi_{2,01}-\frac{2}{r} \xi_{2,0}-\left(\frac{A^{\prime}}{A}\right) \xi_{2,0}=0 \\
\Rightarrow \xi_{2,01} & =\left(\frac{1}{r}+\frac{A^{\prime}}{2 A}\right) \xi_{2,0}
\end{aligned}
$$

Finally, studying the component $\rho_{21,02}$ when $(r A)^{\prime}=1$, we have successively:

$$
\begin{aligned}
d_{0}\left(\xi_{2,12}\right)-d_{2}\left(\xi_{2,01}\right) & =d_{0}\left(-r A \xi_{1,1}\right)-\xi_{1}-\left(\frac{1}{r}+\frac{A^{\prime}}{2 A}\right) \xi_{2,02} \\
& =\frac{r A^{\prime}}{2} \xi_{1,0}+r A\left(\frac{1}{r}+\frac{A^{\prime}}{2 A}\right) \xi_{1,0}-\xi_{1,0} \\
& =\left(A+r A^{\prime}\right) \xi_{1,0}-\xi_{1,0}=0
\end{aligned}
$$

and thus $\xi_{1,0}=0$ cannot be obtained. As we already proved that we had $\xi_{1}=0$ and thus $\xi_{1,1}=0$ but also $\xi_{1,2}=0, \xi_{0,2}=0, \xi_{1,3}=0, \xi_{0,3}=0$, we have therefore obtained $10+5=15$ linearly independent first order equations after only 2 prolongations that can also be obtained by computer algebra in a rather "brute" way.

It follows that one needs one more prolongation in order to obtain $\xi_{1,0}=0$ from $\xi_{1}=0$ by setting $\xi_{1,0}=d_{0} \xi_{1}$ formally.

REMARK 3B.1: We present an alternative approach for finding the same results and illustrate it on two cases. First of all we obtain easily: 


$$
\xi_{0}^{0}+\frac{A^{\prime}}{2 A} \xi^{1}=0, \xi_{1}^{1}-\frac{A^{\prime}}{2 A} \xi^{1}=0, \xi_{2}^{2}+\frac{1}{r} \xi^{1}=0, \xi_{3}^{3}+\cot (\theta) \xi^{2}+\frac{1}{r} \xi^{1}=0
$$

with $\xi_{0}=A \xi^{0}, \xi_{1}=-\frac{1}{A} \xi^{1}, \xi_{2}=-r^{2} \xi^{2}, \xi_{3}=-r^{2} \sin ^{2}(\theta) \xi^{3}$. Then, using $r$ as a summation index, we have:

$$
R_{k l, i j} \equiv \rho_{r l, i j} \xi_{k}^{r}+\rho_{k r, i j} \xi_{l}^{r}+\rho_{k l, r j} \xi_{i}^{r}+\rho_{k l, i r} \xi_{j}^{r}+\xi^{r} \partial_{r} \rho_{k l, i j}=0
$$

and notice that $\rho_{i j}=0 \Rightarrow R_{i j} \equiv \rho_{r j} \xi_{i}^{r}+\rho_{i r} \xi_{j}^{r}+\xi^{r} \partial_{r} \rho_{i j}=0$.

The 6 non-zero components of the Weyl tensor are:

$$
\begin{aligned}
& \rho_{01,01}=+\frac{m}{r^{3}}, \rho_{02,02}=-\frac{m A}{2 r}, \rho_{03,03}=-\frac{m A \sin ^{2}(\theta)}{2 r} \\
& \rho_{12,12}=+\frac{m}{2 r A}, \rho_{13,13}=+\frac{m \sin ^{2}(\theta)}{2 r A}, \rho_{23,23}=-m r \sin ^{2}(\theta)
\end{aligned}
$$

We obtain in particular:

$$
R_{01,01} \equiv 2 \rho_{01,01}\left(\xi_{0}^{0}+\xi_{1}^{1}\right)+\xi^{r} \partial_{r}\left(\rho_{01,01}\right)=\xi^{1} \partial_{1} \rho_{01,01}=0 \Rightarrow \xi^{1}=0
$$

and similarly:

$$
R_{01,02} \equiv \rho_{01,01} \xi_{2}^{1}+\rho_{02,02} \xi_{1}^{2}+\xi^{r} \partial_{r} \rho_{01,02}=\frac{m A}{2 r^{3}}\left(3 \xi_{1,2}-\Omega_{12}\right)=0 \Rightarrow \xi_{2}^{1}=0
$$

and so on, as a way to avoid using computer algebra. However, the main consequence of this remark is to explain the existence of the 15 second order CC. Indeed, denoting by “ ” a linear proportional dependence, we have:

$$
\begin{gathered}
R_{01,01} \sim R_{02,02} \sim R_{03,03} \sim R_{12,12} \sim R_{13,13} \sim R_{23,23} \rightarrow \xi_{1}=0 \\
R_{01,02} \sim R_{13,23} \rightarrow \xi_{1,2}=0 \\
R_{01,03} \sim R_{12,23} \rightarrow \xi_{1,3}=0 \\
R_{01,12} \sim R_{03,23} \rightarrow \xi_{0,2}=0 \\
R_{01,13} \sim R_{02,23} \rightarrow \xi_{0,3}=0 \\
R_{01,23} \rightarrow 0, R_{02,03} \rightarrow 0, R_{02,12} \rightarrow 0, R_{02,13} \rightarrow 0, R_{03,13} \rightarrow 0, R_{12,13} \rightarrow 0
\end{gathered}
$$

as a way to obtain the 5 equalities on the right and thus a total of $20-5=15$ second order CC obtained by elimination. We have to notice that $R_{01,23}=0, R_{02,31}=0 \Rightarrow R_{03,12}=0$ from the identity $R \in \operatorname{ker}(\delta) \Rightarrow R_{01,23}+R_{02,31}+R_{03,12}=0$ and there is no way to have two identical indices in the first jets appearing through the (formal) Lie derivative just described. As for the third order CC, using the equation $\xi_{1,1}=\frac{1}{2} \Omega_{11}-\frac{A^{\prime}}{2 A} \xi_{1}$, we have at least the first prolongations of the previous second order CC to which we have to add the new generating (where the first is the identity $0=0$ ):

$$
d_{0} \xi_{1}-\xi_{1,0}=0, d_{1} \xi_{1}-\xi_{1,1}=0, d_{2} \xi_{1}-\xi_{1,2}=0, d_{3} \xi_{1}-\xi_{1,3}=0, d_{2} \xi_{0,3}-d_{3} \xi_{0,2}=0
$$

provided by the Spencer operator, leading to the crossed terms $d_{i} \xi_{1, j}-d_{j} \xi_{1, i}=0, \forall i, j=1,2,3$ because the Spencer operator is not FI. Finally, we have: 


$$
d_{i} \xi_{1,0}-d_{0} \xi_{1, i} \equiv d_{0}\left(d_{i} \xi_{1}-\xi_{1, i}\right)-d_{i}\left(d_{0} \xi_{1}-\xi_{1,0}\right)=0, \forall i=1,2,3
$$

and do not find any new generating fourth order CC, even if the left member is fourth order. Of course, in each of the preceding situations, we have to replace the jets by their expressions in terms of $j_{2}(\Omega)$ or $j_{3}(\Omega)$ for obtaining the corresponding CC.

Such striking results are brought by the formal Lie derivative of the Weyl tensor because the Ricci tensor vanishes by assumption and we have the splitting Riemann $\simeq$ Ricci $\oplus$ Weyl according to the fundamental diagram II that we discovered as early as in 1988 ([14]) but is still not acknowledged though it can be found in ([15] [21] [23] [24]). In particular, as the Ricci part is vanishing by assumption, we may identify the Riemann part with the Weyl part as tensors ([18] and [24], Th 4.8) and it is possible to prove (using a tedious direct computation or computer algebra) that the only 6 non-zero components are the ones just used in the remark. It is essential to notice that this result bringing a strong condition on the zero jets because of the Lie derivative of the Weyl tensor, thus on the first jets, involves indeed the first derivative of the Weyl tensor because we have a term in $\left(A^{\prime \prime}\right)^{\prime}$. However, as we are dealing with sections, $\xi_{1}=0$ implies $\xi_{1,1}=0$ and we also have $\xi_{0,0}=0, \xi_{1,2}=0, \xi_{1,3}=0$ but not $\xi_{1,0}=0$, these later condition being only brought by another additional prolongation in $R_{1}^{(2)} \subset R_{1}^{(1)}=R_{1}$ and it remains to determine the dimensions of these subsystems, exactly again like in the Macaulay or Janet examples. Knowing that $10=\operatorname{dim}\left(R_{1}\right)=\operatorname{dim}\left(R_{2}\right)>\operatorname{dim}\left(R_{3}\right)=5>\operatorname{dim}\left(R_{4}\right)=4$, we have thus obtained the 16 equations defining $R_{4}$ with $\operatorname{dim}\left(R_{4}\right)=20-16=4$, namely:

$$
\begin{aligned}
& \xi_{3,3}+\sin (\theta) \cos (\theta) \xi_{2}=0 \\
& \xi_{2,3}+\xi_{3,2}-2 \cot (\theta) \xi_{3}=0 \\
& \xi_{1,3}=0 \\
& \xi_{0,3}=0 \\
& \xi_{2,2}=0 \\
& \xi_{1,2}=0 \\
& \xi_{0,2}=0 \\
& \xi_{3,1}-\frac{2}{r} \xi_{3}=0 \\
& \xi_{2,1}-\frac{2}{r} \xi_{2}=0 \\
& \xi_{1,1}=0 \\
& \xi_{0,1}-\frac{A^{\prime}}{A} \xi_{0}=0 \\
& \xi_{3,0}=0 \\
& \xi_{2,0}=0 \\
& \xi_{1,0}=0 \\
& \xi_{0,0}=0 \\
& \xi_{1}=0
\end{aligned}
$$


Setting now $\xi_{0}=A \xi^{0}, \xi_{1}=-\frac{1}{A} \xi^{1}, \xi_{2}=-r^{2} \xi^{2}, \xi_{3}=-r^{2} \xi^{3}$, we may even simplify these equations and get a system not depending on $A$ anymore:

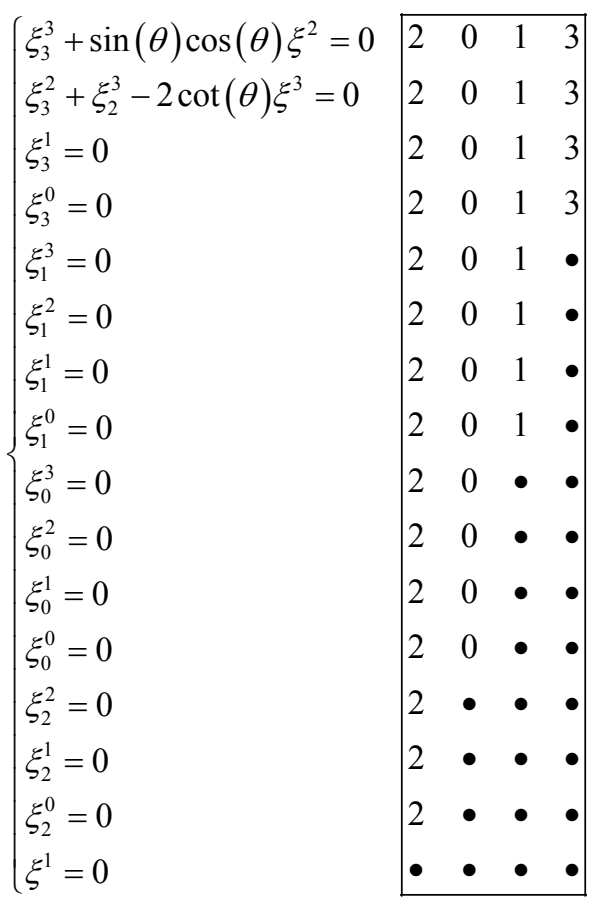

It is easy to check that $R_{1}^{(3)}$, having minimum dimension equal to 4 , is formally integrable, though not involutive as it is finite type, and to exhibit 4 solutions linearly independent over the constants. Indeed, we must have $\xi^{0}=c$ where $c$ is a constant and we may drop the time variable not appearing elsewhere while using the equation $\xi^{1}=0$. It follows that $\xi^{2}=f(\theta, \phi), \xi^{3}=g(\theta, \phi)$ while $f, g$ are solutions of the first, second and fifth equations of Killing type with a general solution depending on 3 constants, a result leading to an elementary problem of 2-dimensional elasticity left to the reader as an exercise. The system $R_{1}^{(3)}$ is formally integrable while the system $R_{2}^{(2)}$ is involutive. Having in mind the PP procedure, it follows that the CC are of order 2, 3 and 4 along the following commutative and exact diagram and its various prolongations:

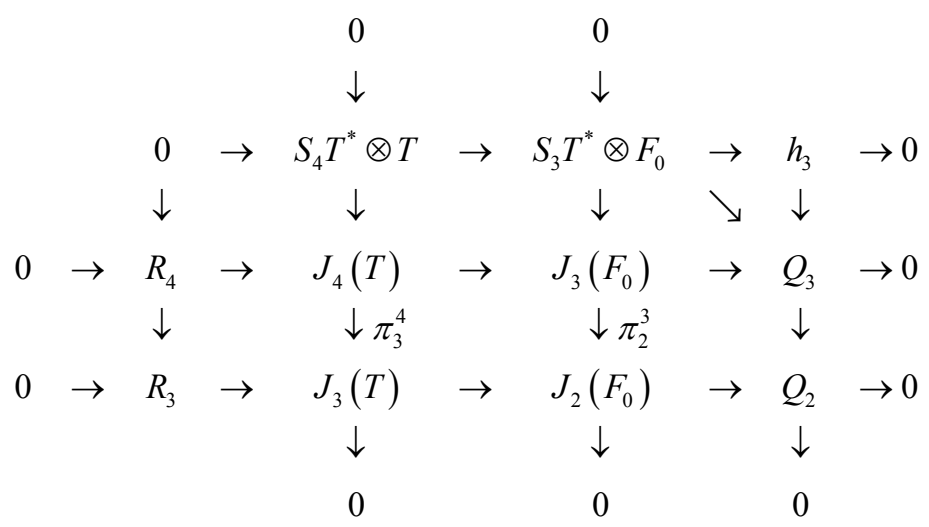




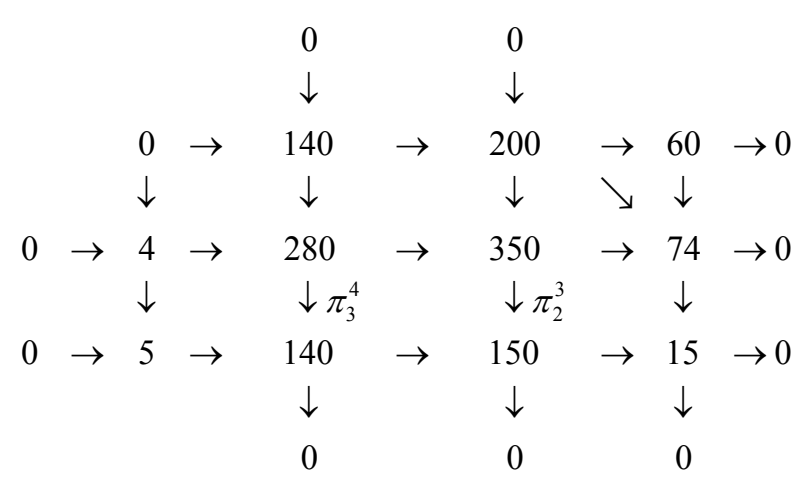

and its various prolongations like:

$$
\begin{aligned}
& \begin{array}{lll}
0 & 0 & 0 \\
\downarrow & \downarrow & \downarrow
\end{array} \\
& 0 \rightarrow S_{5} T^{*} \otimes T \rightarrow S_{4} T^{*} \otimes F_{0} \rightarrow h_{4} \quad \rightarrow 0 \\
& \downarrow \quad \downarrow \quad \downarrow \quad \searrow \quad \downarrow
\end{aligned}
$$

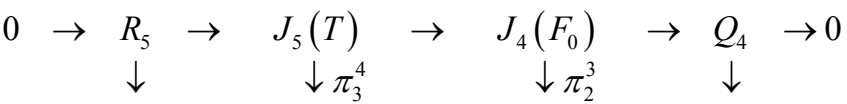

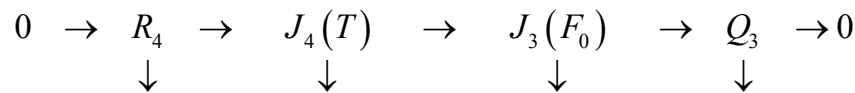

$$
\begin{aligned}
& 0 \quad 0 \quad 0 \quad 0 \\
& \begin{array}{lll}
0 & 0 & 0
\end{array} \\
& \downarrow \quad \downarrow \quad \downarrow
\end{aligned}
$$

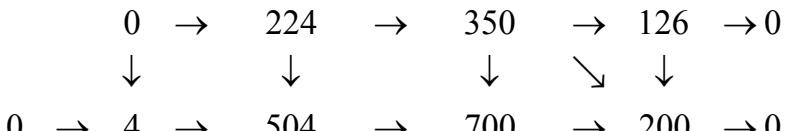

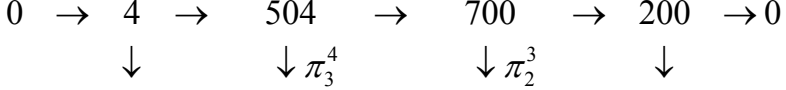

$$
\begin{aligned}
& 0 \rightarrow 4 \rightarrow 280 \rightarrow 350 \rightarrow 74 \rightarrow 0 \\
& \begin{array}{llll}
\downarrow & \downarrow & \downarrow & \downarrow \\
0 & 0 & 0 & 0
\end{array}
\end{aligned}
$$

If we define $F_{1}=Q_{4}$, we have the exact sequences with $\operatorname{dim}\left(R_{3+r}\right)=\operatorname{dim}\left(R_{3}\right)=4, \forall r \geq 0$ :

$$
0 \rightarrow R_{5+r} \rightarrow J_{5+r}(T) \rightarrow J_{4+r}\left(F_{0}\right) \rightarrow J_{r}\left(F_{1}\right)
$$

by applying the Spencer $\delta$-map inductively to the symbol sequences:

$$
0 \rightarrow S_{5+r} T^{*} \otimes T \rightarrow S_{4+r} T^{*} \otimes F_{0} \rightarrow S_{r} T^{*} \otimes F_{1}
$$

and chasing as usual along the south-west to north-east diagonal. However, exactly like in the Macaulay example 2A.9 where we needed 2 prolongations while here we need 3 prolongations, we could also define $F_{1}=Q_{3}$. Indeed, applying the Spencer operator $d$ of Proposition 2C.3 like in ([12], p. 190) or ([13], p. 688), the local exactnes of the sequence $T \rightarrow F_{0} \rightarrow F_{1}$ is equivalent to the local exactness of the first Spencer sequence $R_{4} \stackrel{d}{\longrightarrow} T^{*} \otimes R_{3} \stackrel{d}{\longrightarrow} \wedge^{2} T^{*} \otimes R_{2}$. As the second Spencer sequence is locally isomorphic to the tensor product of 
the Poincaré sequence by a 4-dimensional Lie algebra, it is locally exact and the corresponding first Spencer sequence $R_{5} \stackrel{d}{\longrightarrow} T^{*} \otimes R_{4} \stackrel{d}{\longrightarrow} \wedge^{2} T^{*} \otimes R_{3}$ is also locally exact. By projection, it is thus sufficient to prove the injectivity of $d$ in the sequence $0 \rightarrow T^{*} \otimes\left(R_{3} / R_{3}^{(1)}\right) \stackrel{d}{\longrightarrow} \wedge^{2} T^{*} \otimes\left(R_{2} / R_{2}^{(1)}\right)$. A tricky computation, justifying the use of the Spencer operator in Remark 3B.1, finally allows to construct inductively the simplest, shortest, formally and locally exact differential sequence:

$$
0 \rightarrow \Theta \rightarrow 4 \underset{1}{\longrightarrow} 10 \underset{3}{\longrightarrow} 74 \underset{1}{\longrightarrow} 170 \underset{1}{\longrightarrow} 164 \underset{1}{\longrightarrow} 76 \underset{1}{\longrightarrow} 14 \rightarrow 0
$$

However, this fact is of no importance compared to the following comments that we now provide and will be explained later on in a much simpler direct way.

First of all, denoting by $R_{2}^{\prime}=R_{2}^{(1)} \subset R_{2}$ with $\operatorname{dim}\left(R_{2}^{\prime}\right)=4$ the involutive system provided by the PP procedure, we are in position to construct the corresponding canonical/involutive (lower) Janet and (upper) Spencer sequences along the following fundamental diagram $I$ already constructed in many books and papers (In particular, we advise the curious reader to look at the very striking Example 3.14 described in [17], p. 119 and showing the importance of involution) and presented in the last subsection $A$. In this diagram, not depending any longer on $m$, we have now $C_{r}=\wedge^{r} T^{*} \otimes R_{2}^{\prime}$ and provide the fiber dimensions below:

$$
\begin{aligned}
& \begin{array}{lllll}
0 & 0 & 0 & 0 & 0 \\
\downarrow & \downarrow & \downarrow & \downarrow & \downarrow
\end{array}
\end{aligned}
$$

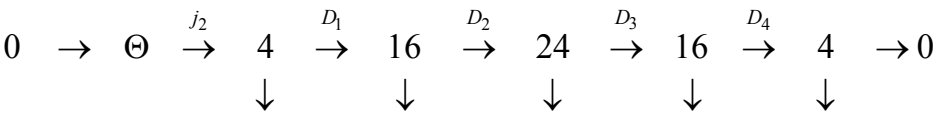

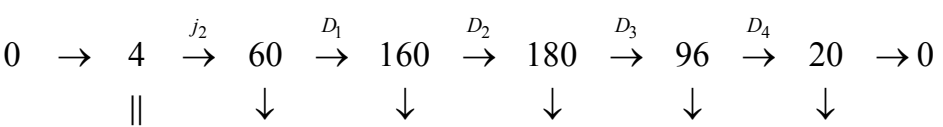

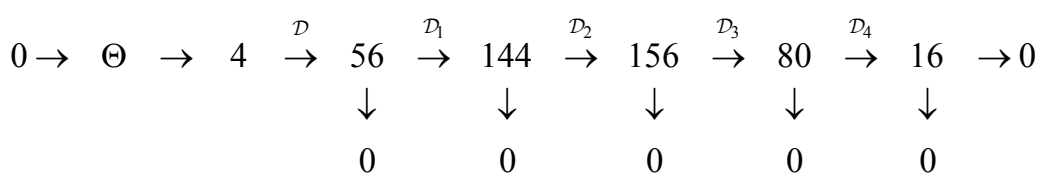

We notice the vanishing of the Euler-Poincaré characteristics:

$$
\begin{aligned}
& 4-16+24-16+4=0, \\
& 4-60+160-180+96-20=0, \\
& 4-56+144-156+80-16=0
\end{aligned}
$$

In actual practice, all the preceding computations have been finally used to reduce the Poincaré group to its subgroup made with only one time translation and three space rotations!. On the contrary, we have proved during the last forty years that one must increase the Poincaré group (10 parameters), first to the Weyl group (11 parameters by adding 1 dilatation) and finally to the conformal group of space-time (15 parameters by adding 4 elations) as in ([41]), while only dealing with the Spencer sequence in order to increase the dimensions of the Spencer bundles and thus the number of corresponding potentials and fields. In 
view of the size of the matrices involved, we wish therefore good luck to the reader who should like to find back these results by using computer algebra!

Now, in order to convince the reader that only new methods can allow to study the strange phenomena happening in the constructions of CC (high order, sudden increase in the number of generators,...), we shall turn over totally the previous approach and use a totally different point of view in order to shortcut the use of computer algebra, having in mind that we already know the final formally integrable system $R_{3} \subset J_{3}(T)$ with $\operatorname{dim}\left(R_{3}\right)=4$ but the same method could be used for other cases. For this, we use the known Killing vector $\partial_{t}$ and the zero order equation $\xi^{1}=0$ in order to restrict $T$ to a sub-vector bundle $E \subset T$ of dimension 2 with section $\left(\xi^{2}, \xi^{3}\right)$ in order to have a first order system with 3 independent variables $(r, \theta, \phi)=(1,2,3)$ and 2 unknowns, obtained by eliminating $\xi^{1}$ and $\xi^{0}$ as follows in order to get an equivalent system for $\xi^{2}, \xi^{3}$ for the variables $(1,2,3)$ with only 5 equations:

$$
\left\{\begin{array}{l}
\xi_{3}^{3}+\sin (\theta) \cos (\theta) \xi^{2}=0 \\
\xi_{3}^{2}+\xi_{2}^{3}-2 \cot (\theta) \xi^{3}=0 \\
\xi_{1}^{3}=0 \\
\xi_{1}^{2}=0 \\
\xi_{2}^{2}=0
\end{array}\right.
$$

As a byproduct, we have the following commutative diagrams:

$$
\begin{aligned}
& 0 \\
& \downarrow \quad \downarrow \\
& 0 \quad \rightarrow \quad S_{3} T^{*} \otimes E \quad \rightarrow \quad S_{2} T^{*} \otimes F_{0} \quad \rightarrow \quad h_{2} \quad \rightarrow 0 \\
& \downarrow \delta \quad \downarrow \delta \quad \downarrow \\
& 0 \quad \rightarrow \quad T^{*} \otimes S_{2} T^{*} \otimes E \quad \rightarrow \quad T^{*} \otimes T^{*} \otimes F_{0} \quad \rightarrow \quad T^{*} \otimes Q_{1} \rightarrow 0 \\
& \downarrow \delta \quad \downarrow \delta \quad \downarrow \\
& 0 \rightarrow \wedge^{2} T^{*} \otimes g_{1} \rightarrow \wedge^{2} T^{*} \otimes T^{*} \otimes E \quad \rightarrow \quad \wedge^{2} T^{*} \otimes F_{0} \quad \rightarrow \quad 0 \\
& \downarrow \delta \quad \downarrow \delta \quad \downarrow \\
& 0 \rightarrow \wedge^{3} T^{*} \otimes E \quad=\quad \wedge^{3} T^{*} \otimes E \quad \rightarrow \quad 0
\end{aligned}
$$

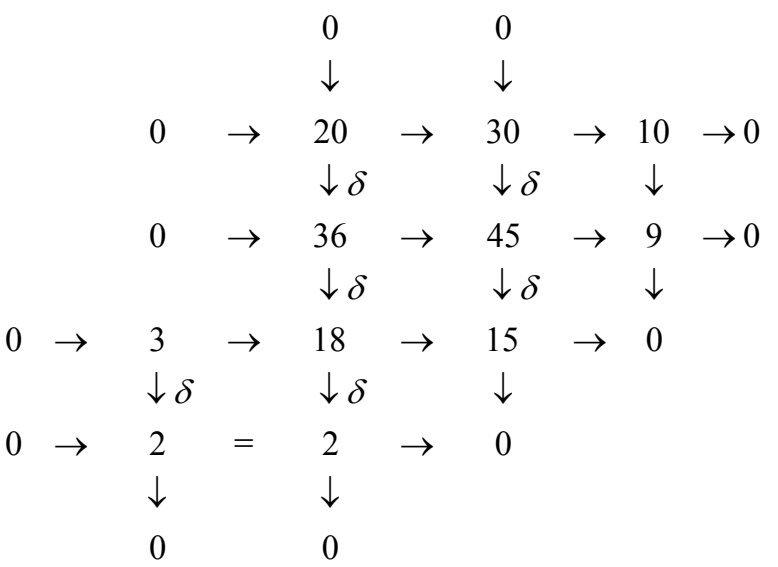


The next result points out the importance of the Spencer $\delta$-cohomology and will be justified later on in cartesian coordinates as it is intrinsic:

LEMMA 3B.2: The last symbol diagram is commutative and exact. In particular, the lower left map $\delta$ is surjective and thus the upper right induced map $h_{2} \rightarrow T^{*} \otimes Q_{1}$ is also surjective while these two maps have isomorphic kernels.

Proof: The 3 components of $\wedge^{2} T^{*} \otimes g_{1}$ are $\left\{v_{2,12}^{3}, v_{2,13}^{3}, v_{2,23}^{3}\right\}$ and the kernel of the map $\delta$ is described by the two linear equations:

$$
v_{1,23}^{2}+v_{2,31}^{2}+v_{3,12}^{2}=0, \quad v_{1,23}^{3}+v_{2,31}^{3}+v_{3,12}^{3}=0
$$

that is to say by the two linearly independent equations:

$$
v_{2,12}^{3}=0, \quad v_{2,13}^{3}=0
$$

Accordingly, in the left column we have:

$$
\operatorname{dim}\left(H^{2}\left(g_{1}\right)\right)=\operatorname{dim}\left(Z^{2}\left(g_{1}\right)\right)=\operatorname{dim}(\operatorname{ker}(\delta))=1
$$

An unusual snake-type diagonal chase left to the reader as an exercise proves that the induced map $h_{2} \rightarrow T^{*} \otimes Q_{1}$ is surjective with a kernel isomorphic to $H^{2}\left(g_{1}\right)$. This is a crucial result because it also proves that the additional CC has only to do with the the single second order component of the Riemann tensor in dimension 2, a striking result that could not even be imagined by standard methods.

Q.E.D.

Now, we have explained why the new zero order PD equation $\xi_{1}=0 \Leftrightarrow \xi^{1}=0$ should be replaced by the condition $x^{i} \xi_{i}=0$ by using the space euclidan metric for lowering the indices. Differentiating with respect to $x^{j}$, we obtain $\delta_{j}^{i} \xi_{i}+x^{i} \xi_{i, j}=0$ with the Kronecker symbol $\delta$ and, contracting with $x^{j}$ we finally get $x^{j} \xi_{j}+x^{i} x^{j} \xi_{i, j}=0$. hence on space, we get a new subsystem by adding to the standard Killing system of space $(n=3)$ the above zero order constraint in order to get a system $R_{1}^{\prime}$ with $\operatorname{dim}\left(R_{1}^{\prime}\right)=(3+9)-(6+2+1)=3$. Coming back to the computation previously done with the Schwarzschild metric while using only $\xi^{0}$ and $\xi^{1}$, we discover that the new system does not any longer depend on " $A$ " (See other examples in [16]).

Its study can be therefore replaced by that of the 3-dimensional system $R_{1}^{\prime}$ which is defining a nontransitive system of infinitesimal lie equations, that is the map $\pi_{0}^{1}: R_{1}^{\prime} \rightarrow T$ is no longer surjective, a result modifying the constructions of the Vessiot structure equations but this is out of our story. Suppressing from now on the "'” for simplicity, we are thus led to $n=3$ and the formally integrable system of 9 linearly independent equations where $\omega$ is the Euclidean metric:

$$
\xi_{i, j}+\xi_{j, i}=0, \quad x^{i} \xi_{i, j}+\xi_{j}=0, \quad x^{i} \xi_{i}=0
$$


because $x^{j}\left(x^{i} \xi_{i, j}+\xi_{j}\right)=x^{i} x^{j} \xi_{i, j}+x^{i} \xi_{i}=x^{i} \xi_{i}=0$.

Changing slighty the notations with now $n=3, m=\operatorname{dim}(E)=2$ in order to keep an upper index for any unknown while setting $\xi^{3}=-\frac{x^{1}}{x^{3}} \xi^{1}-\frac{x^{2}}{x^{3}} \xi^{2}$, we get the following system $R_{1} \subset J_{1}(E)$ with $\operatorname{dim}\left(R_{1}\right)=3$ because $\operatorname{par}_{1}=\left\{\xi^{1}, \xi^{2}, \xi_{1}^{2}\right\}$ and corresponding Janet tabular:

$$
\left\{\begin{array}{l|l|ll}
\Phi^{5} \equiv \xi_{3}^{2}+\frac{x^{1}}{x^{3}} \xi_{1}^{2}-\frac{1}{x^{3}} \xi^{2}=0 & 1 & 2 & 3 \\
\Phi^{4} \equiv \xi_{3}^{1}-\frac{x^{2}}{x^{3}} \xi_{1}^{2}-\frac{1}{x^{3}} \xi^{1}=0 & 1 & 2 & 3 \\
\Phi^{3} \equiv \xi_{2}^{2}=0 \\
\Phi^{2} \equiv \xi_{2}^{1}+\xi_{1}^{2}=0=0 \\
\Phi^{1} \equiv \xi_{1}^{1}=0
\end{array} \mid \begin{array}{lll}
1 & 2 & \bullet \\
1 & 2 & \bullet
\end{array}\right.
$$

It is easy to check that all the second order jets vanish and that the general solution $\left\{\xi^{1}=a x^{2}+b x^{3}, \xi^{2}=-a x^{2}+c x^{3}\right\}$ depends on 3 arbitrary constants $(a, b, c)$ in such a way that the three space rotations are separately and respectively obtained by each element of the basis $\{(1,0,0),(0,1,0),(0,0,1)\}$.

As before but with a different system, we have the following commutative diagrams:

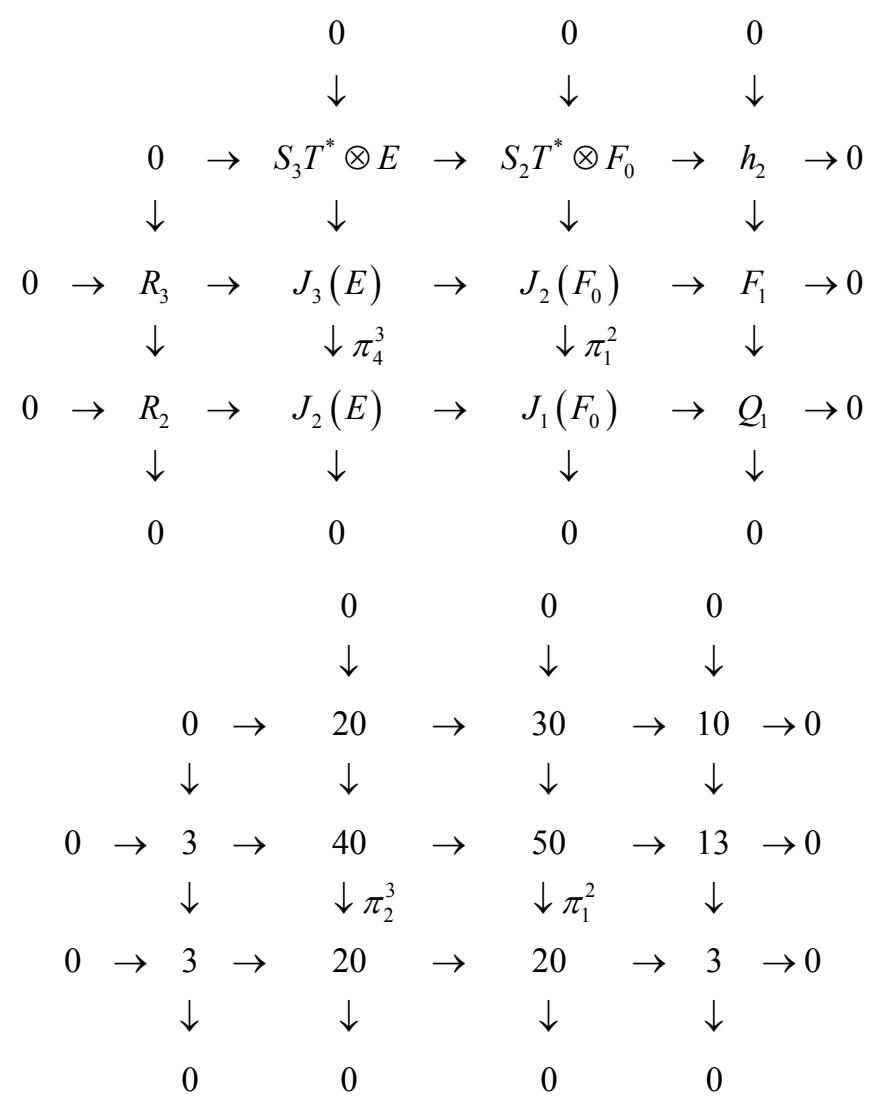




$$
\begin{aligned}
& 0 \quad 0 \\
& \downarrow \quad \downarrow \\
& 0 \quad \rightarrow \quad S_{3} T^{*} \otimes E \quad \rightarrow \quad S_{2} T^{*} \otimes F_{0} \quad \rightarrow \quad h_{2} \quad \rightarrow 0 \\
& \downarrow \delta \quad \downarrow \delta \\
& 0 \quad \rightarrow \quad T^{*} \otimes S_{2} T^{*} \otimes E \quad \rightarrow \quad T^{*} \otimes T^{*} \otimes F_{0} \quad \rightarrow \quad T^{*} \otimes Q_{1} \quad \rightarrow 0 \\
& \downarrow \delta \quad \downarrow \delta \quad \downarrow \\
& 0 \rightarrow \wedge^{2} T^{*} \otimes g_{1} \rightarrow \wedge^{2} T^{*} \otimes T^{*} \otimes E \rightarrow \wedge^{2} T^{*} \otimes F_{0} \quad \rightarrow \quad 0 \\
& \downarrow \delta \quad \downarrow \delta \quad \downarrow \\
& 0 \rightarrow \wedge^{3} T^{*} \otimes E \quad=\quad \wedge^{3} T^{*} \otimes E \quad \rightarrow \quad 0 \\
& \downarrow \quad \downarrow \\
& 0 \quad 0
\end{aligned}
$$

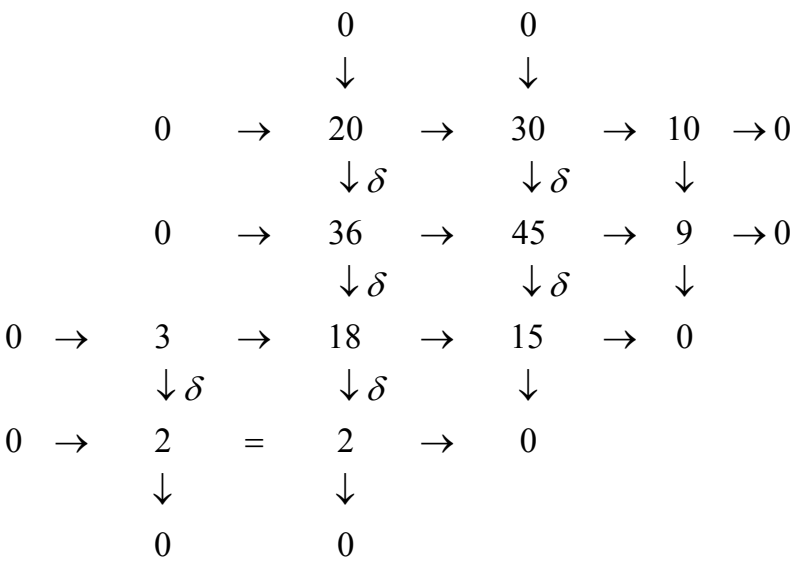

The next result points out the importance of the Spencer $\delta$-cohomology:

LEMMA 3B.3: The last symbol diagram is commutative and exact. In particular, the lower left map $\delta$ is surjective and thus the upper right induced map $h_{2} \rightarrow T^{*} \otimes Q_{1}$ is also surjective while these two maps have isomorphic kernels.

Proof: The 3 components of $\wedge^{2} T^{*} \otimes g_{1}$ are $\left\{v_{1,12}^{2}, v_{1,13}^{2}, v_{1,23}^{2}\right\}$ and the map $\delta$ is described by the two linear equations:

$$
v_{1,23}^{1}+v_{2,31}^{1}+v_{3,12}^{1}=0, \quad v_{1,23}^{2}+v_{2,31}^{2}+v_{3,12}^{2}=0
$$

that is to say by the two linearly independent equations:

$$
v_{1,13}^{2}+\frac{x^{2}}{x^{3}} v_{1,12}^{2}=0, \quad v_{1,23}^{2}-\frac{x^{1}}{x^{3}} v_{1,12}^{2}=0
$$

Accordingly, in the left column we have:

$$
\operatorname{dim}\left(H^{2}\left(g_{1}\right)\right)=\operatorname{dim}\left(Z^{2}\left(g_{1}\right)\right)=\operatorname{dim}(\operatorname{ker}(\delta))=1
$$

An unusual snake-type diagonal chase left to the reader as an exercise proves that the induced map $h_{2} \rightarrow T^{*} \otimes Q_{1}$ is surjective with a kernel isomorphic to $H^{2}\left(g_{1}\right)$. This is indeed a crucial result because it also proves that the additional CC has only to do with the the single second order component of the Riemann tensor in dimension 2, a striking result that could not even be imagined by standard methods.

Q.E.D. 
Collecting the above results, we find the 3 first order differentially independent generating CC coming from the Janet tabular and the additional single second order generating CC describing the 2-dimensional Riemann operator, that is the linearized Riemann tensor in the space $\left(x^{1}, x^{2}\right)$ :

$$
\left\{\begin{array}{l}
\Psi^{4} \equiv d_{22} \Phi^{1}+d_{11} \Phi^{3}-d_{12} \Phi^{2}=0 \\
\Psi^{3} \equiv d_{3} \Phi^{3}-d_{2} \Phi^{5}+\frac{x^{1}}{x^{3}} d_{1} \Phi^{3}-\frac{1}{x^{3}} \Phi^{3}=0 \\
\Psi^{2} \equiv d_{3} \Phi^{2}-d_{2} \Phi^{4}-d_{1} \Phi^{5}-\frac{x^{2}}{x^{3}} d_{1} \Phi^{3}+\frac{x^{1}}{x^{3}}\left(d_{1} \Phi^{2}-d_{2} \Phi^{1}\right)-\frac{1}{x^{3}} \Phi^{2}=0 \\
\Psi^{1} \equiv d_{3} \Phi^{1}-d_{1} \Phi^{4}-\frac{x^{2}}{x^{3}}\left(d_{1} \Phi^{2}-d_{2} \Phi^{1}\right)-\frac{1}{x^{3}} \Phi^{1}=0
\end{array}\right.
$$

An elementary but tedious computation provides the second order CC:

$$
x^{3}\left(d_{22} \Psi^{1}+d_{11} \Psi^{3}-d_{12} \Psi^{2}\right)-\left(x^{1} d_{1} \Psi^{4}+x^{2} d_{2} \Psi^{4}+x^{3} d_{3} \Psi^{4}\right)-2 \Psi^{4}=0
$$

The corresponding differential sequence written with differential modules over the ring $D=K\left[d_{1}, d_{2}, d_{3}\right]$ with $K=\mathbb{Q}\left(x^{1}, x^{2}, x^{3}\right)$ is:

$$
0 \rightarrow D \underset{2}{\longrightarrow} D^{4} \underset{2}{\longrightarrow} D^{5} \underset{1}{\longrightarrow} D^{2} \stackrel{p}{\longrightarrow} M \rightarrow 0
$$

where $p$ is the canonical (residual) projection. We check indeed that $1-4+5-2=0$ but this sequence is quite far from being even strictly exact. Of course, as $R_{2}$ is involutive, we may set $C_{r}=\wedge^{r} T^{*} \otimes R_{2}$ and obtain the corresponding canonical second Spencer sequence which is induced by the Spencer operator:

$$
0 \rightarrow \Theta \stackrel{j_{2}}{\longrightarrow} C_{0} \stackrel{D_{1}}{\longrightarrow} C_{1} \stackrel{D_{2}}{\longrightarrow} C_{2} \stackrel{D_{3}}{\longrightarrow} C_{3} \rightarrow 0
$$

with dimensions:

$$
0 \rightarrow \Theta \stackrel{j_{2}}{\longrightarrow} 3 \stackrel{D_{1}}{\longrightarrow} 9 \stackrel{D_{2}}{\longrightarrow} 9 \stackrel{\stackrel{D_{3}}{\longrightarrow}}{\longrightarrow} 3 \rightarrow 0
$$

We let the reader compute the corresponding Janet sequence as a first step towards the Vessiot structure equations which are not easily obtained because we have now:

$$
x^{i} \xi_{i}=x^{i} \omega_{i j} \xi^{j} \Rightarrow \xi_{i, j}+\xi_{j, i}-2 \gamma_{i j}^{r} \xi_{r}=0
$$

where $\gamma$ denotes the Christoffel symbols.

This result justifies "a fortiori" the comments we have already provided. The reader may compare such an example with the Janet example (where we have one third order CC and one sixth order additional CC) with the major difference that we have now a formally integrable system. We do not know any other similar situation.

In order to achieve the study of the Schwarzschild metric in a purely intrinsic way, we prove that the situation of the previous example is just describing the way to exhibit the torsion part $t(M) \subseteq M$ of a differential module by computing a certain extension module. Coming back to the systems already obtained and keeping in mind that 
$\xi_{1}=0 \Rightarrow \xi_{0,0}=0, \xi_{0,1}-\frac{A^{\prime}}{A} \xi_{0}=0, \xi_{0,2}=0, \xi_{0,3}=0 \Rightarrow \xi^{0}=c s t \quad$ while replacing $r^{2} \xi_{2}, r^{2} \xi_{3}$ by $\xi^{2}, \xi^{3}$ respectively, we may therefore replace the integration of the previous system by that of the simpler system:

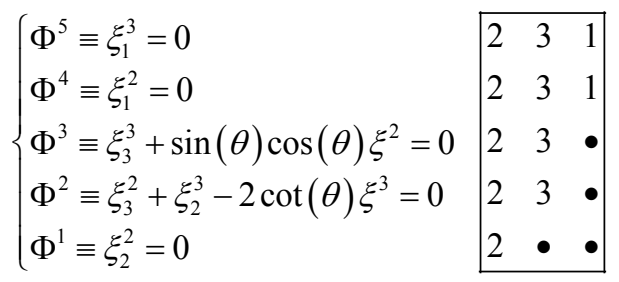

allowing to define an isomorphic differential module because both systems are formally integrable though not involutive, with the same dimension $2+(2 \times 3)-5=3$ with $\operatorname{par}_{2}=\operatorname{par}_{1}=\left\{\xi^{2}, \xi^{3}, \xi_{2}^{3}\right\}$. We have now similarly the 3 first order $\mathrm{CC}$ and the single second order $\mathrm{CC}$ :

$$
\left\{\begin{aligned}
\Psi^{4} \equiv & d_{33} \Phi^{1}+d_{22} \Phi^{3}-d_{23} \Phi^{2}-\sin (\theta) \cos (\theta) d_{2} \Phi^{1} \\
& -2 \cot (\theta) d_{2} \Phi^{3}-2 \sin ^{2}(\theta) \Phi^{1}+\frac{2}{\sin ^{2}(\theta)} \Phi^{3}=0 \\
\Psi^{3} \equiv & d_{1} \Phi^{3}-d_{3} \Phi^{5}-\sin (\theta) \cos (\theta) \Phi^{4}=0 \\
\Psi^{2} \equiv & d_{1} \Phi^{2}-d_{3} \Phi^{4}-d_{2} \Phi^{5}-2 \cot (\theta) \Phi^{5}=0 \\
\Psi^{1} \equiv & d_{1} \Phi^{1}-d_{2} \Phi^{4}=0
\end{aligned}\right.
$$

describing again the single component of the linearized Riemann tensor for $(\theta, \phi)$ and the first Spencer cohomology group $H^{2}\left(g_{1}\right)$ of the first symbol $g_{1} \subset T^{*} \otimes E$ with $\operatorname{dim}(E)=2$ and $\operatorname{dim}\left(H^{2}\left(g_{1}\right)\right)=\operatorname{dim}\left(\wedge^{2} T^{*} \otimes g_{1}\right)-\operatorname{dim}\left(\wedge^{3} T^{*} \otimes E\right)=3-2=1$. Of course, we could even simplified the later system by considering the new system:

$$
\left\{\begin{array}{l}
\Phi^{3} \equiv \xi_{3}^{3}+\sin (\theta) \cos (\theta) \xi^{2}=0 \\
\Phi^{2} \equiv \xi_{3}^{2}+\xi_{2}^{3}-2 \cot (\theta) \xi^{3}=0 \\
\Phi^{1} \equiv \xi_{2}^{2}=0
\end{array}\right.
$$

We let the reader fill in the details and discover again the only CC $\Psi^{4}=\Psi=0$.

Considering both situations already studied with $n=3, m=2$, we discover that the differential modules defined by the system $\Psi^{1}=0, \Psi^{2}=0, \Psi^{3}=0$ are isomoprphic, provided we extend conveniently the ground differential field. Hence, in both cases, we have $t(M) \neq 0$ and the torsion submodule has a single generator namely the residue of $\Psi^{4}$ which is satisfying for example the so-called autonomous equation:

$$
x^{1} d_{1} \Psi^{4}+x^{2} d_{2} \Psi^{4}+x^{3} d_{3} \Psi^{4}+2 \Psi^{4}=0
$$

Setting $M^{\prime}=M / t(M)$, we have the short exact sequence:

$$
0 \rightarrow t(M) \rightarrow M \rightarrow M^{\prime} \rightarrow 0
$$

where the torsion-free differential module $M^{\prime}$ is now defined by 
$\Psi^{1}=0, \cdots, \Psi^{4}=0$. The explanation can only be natural in the framework of differential homological algebra because, by construction, the CC operator $D_{1}$ is parametrized by $D$ and MUST therefore provide the torsion-free differential module $M^{\prime}$. This result explains for the fiirst time the intrinsic character of the additional higher order generating CC that can be found in an apparently strange manner. In a more detailed way, let us proceed as follows in order to construct the following commutative diagram:

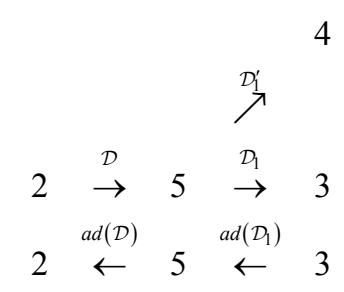

1) Write down the operator $\mathcal{D}_{1}:\left(\Phi^{1}, \cdots, \Phi^{5}\right) \rightarrow\left(\Psi^{1}, \Psi^{2}, \Psi^{3}\right)$ as we did.

2) Multiply by test functions $\lambda=\left(\lambda^{1}, \lambda^{2}, \lambda^{3}\right)$, integrate by parts and construct the formal adjoint $\operatorname{ad}\left(\mathcal{D}_{1}\right):\left(\lambda^{1}, \lambda^{2}, \lambda^{3}\right) \rightarrow\left(\mu^{1}, \cdots, \mu^{5}\right)$ :

$$
\left\{\begin{array}{l}
-d_{1} \lambda^{1}=\mu^{1} \\
-d_{1} \lambda^{2}=\mu^{2} \\
-d_{1} \lambda^{3}=\mu^{3} \\
d_{3} \lambda^{2}+d_{2} \lambda^{1}-\sin (\theta) \cos (\theta) \lambda^{3}=\mu^{4} \\
d_{3} \lambda^{3}+d_{2} \lambda^{2}-2 \cos (\theta) \lambda^{2}=\mu^{5}
\end{array}\right.
$$

3) Construct generating CC as an operator $\operatorname{ad}(\mathcal{D}):\left(\mu^{1}, \cdots, \mu^{5}\right) \rightarrow\left(v^{1}, v^{2}\right)$ :

$$
\left\{\begin{array}{l}
d_{1} \mu^{4}+d_{3} \mu^{2}+d_{2} \mu^{1}-\sin (\theta) \cos (\theta) \mu^{3}=v^{1} \\
d_{1} \mu^{5}+d_{3} \mu^{3}+d_{2} \mu^{2}-2 \cos (\theta) \mu^{2}=v^{2}
\end{array}\right.
$$

4) Exhibit $\operatorname{ad}(\operatorname{ad}(\mathcal{D}))=\mathcal{D}:\left(\Upsilon^{1}, \Upsilon^{2}\right) \rightarrow\left(\Phi^{1}, \cdots, \Phi^{5}\right)$ :

$$
\left\{\begin{array}{l}
-d_{2} \Upsilon^{1}=\Phi^{1} \\
-d_{3} \Upsilon^{1}-d_{2} \Upsilon^{2}-2 \cos (\theta) \Upsilon^{2}=\Phi^{2} \\
-d_{3} \Upsilon^{2}-\sin (\theta) \cos (\theta) \Upsilon^{1}=\Phi^{3} \\
-d_{1} \Upsilon^{1}=\Phi^{4} \\
-d_{1} \Upsilon^{2}=\Phi^{5}
\end{array}\right.
$$

5) Construct generating CC $\mathcal{D}^{\prime}:\left(\Phi^{1}, \cdots, \Phi^{5}\right) \rightarrow\left(\Psi^{1}, \Psi^{2}, \Psi^{3}, \Psi^{4}\right)$ and compare $\mathcal{D} \leq \mathcal{D}^{\prime}$. Then each new CC is a torsion element of the differential module determined by $\mathcal{D}$ which is thus parametrized by $\mathcal{D}_{-1}$ if and only if $\mathcal{D}^{\prime}=\mathcal{D}$. In the present case, $\Psi^{1}, \Psi^{2}, \Psi^{3}$ being differentially independent, we find the only additional generating CC $\Psi^{4}=0$.

Accordingly, the situation in GR cannot evolve as long as people will not acknowledge the fact that the components of the Weyl tensor are similarly playing the part of torsion elements (the so-called Lichnerowicz waves in [42] 
[43]) for the equations $R i c c i=0$ for reasons only depending on the group structure of the conformal group of space-time and bring the splitting Riemann $=$ Weyl $\oplus$ Ricci.

C) KERR METRIC:

We now write the Kerr metric in Boyer-Lindquist coordinates:

$$
\begin{aligned}
d s^{2}= & \frac{\rho^{2}-m r}{\rho^{2}} d t^{2}-\frac{\rho^{2}}{\Delta} d r^{2}-\rho^{2} d \theta^{2}+\frac{2 a m r \sin ^{2}(\theta)}{\rho^{2}} d t d \phi \\
& -\frac{\left(r^{2}+a^{2}\right)^{2}-a^{2} \Delta \sin ^{2}(\theta)}{\rho^{2}} \sin ^{2}(\theta) d \phi^{2}
\end{aligned}
$$

where we have set $\Delta=r^{2}-m r+a^{2}, \rho^{2}=r^{2}+a^{2} \cos ^{2}(\theta)$ and we check that:

$$
a=0 \Rightarrow d s^{2}=\left(1-\frac{m}{r}\right) d t^{2}-\frac{1}{1-\frac{m}{r}} d r^{2}-r^{2} d \theta^{2}-r^{2} \sin (\theta)^{2} d \phi^{2}
$$

as a well-known way to recover the Schwarschild metric. Now, we notice that $t$ or $\phi$ do not appear in the coefficients of the metric and thus, as the maximum subgroup of invariance of the Kerr metric must be contained in the maximum subgroup of invariance of the Schwarzschild metric because of the above limit when $a \rightarrow 0$, we obtain the only possible 2 infinitesimal generators $\left\{\partial_{t}, \partial_{\phi}\right\}$ and we have the fundamental diagram I with fiber dimensions:

$$
\begin{aligned}
& \begin{array}{lllll}
0 & 0 & 0 & 0 & 0 \\
\downarrow & \downarrow & \downarrow & \downarrow & \downarrow
\end{array}
\end{aligned}
$$

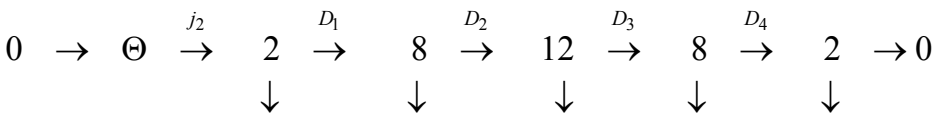

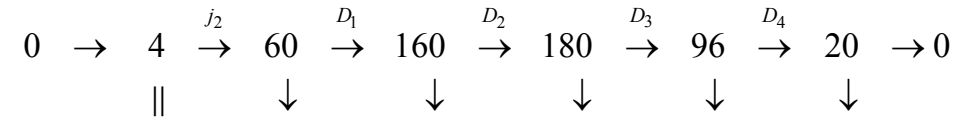

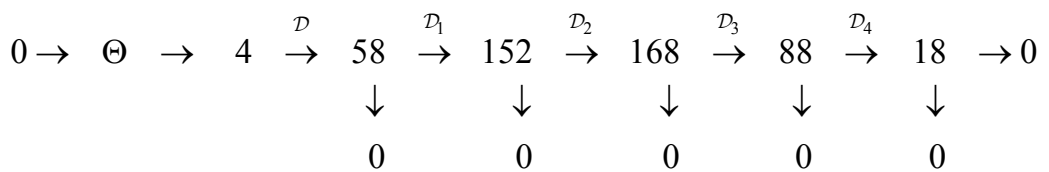

with Euler-Poincaré characteristic 4-58+152-168+88-18=0. Comparing the surprisingly high dimensions of the Janet bundles with the surprisingly low dimensions of the Spencer bundles needs no comment on the physical usefulness of the Janet sequence, despite its purely mathematical importance. In addition, using now the same notations as in the preceding section, we have the additional zero order equations $\xi^{1}=0, \xi^{2}=0$ produced by the non-zero components of the Weyl tensor and thus, at best,

$\operatorname{dim}\left(R_{0}^{(3)}\right)=2 \Leftrightarrow \operatorname{dim}\left(R_{1}^{(2)}\right)=2$, if the zero order equations are obtained after only two prolongations. As these zero order equations depend on $j_{2}(\Omega)$, at best, we should obtain therefore eventually $\operatorname{dim}\left(Q_{2}\right)=10+2=12$ CC of order 2 and $60-(4 \times 12)=12$ CC of order 3 at least. 
Using finally cartesian coordinates with $\xi^{3}=0, x^{1} \xi^{1}+x^{2} \xi^{2}=0$, we have only to study the following first order involutive system for $\xi^{1}=\xi$ with coefficients no longer depending on $(a, m)$, providing the only generator $x^{1} \partial_{2}-x^{2} \partial_{1}$

$$
\begin{aligned}
& \left\{\begin{array}{l}
\Phi^{3} \equiv \xi_{3}=0 \\
\Phi^{2} \equiv \xi_{2}-\frac{1}{x^{2}} \xi=0 \\
\Phi^{1} \equiv \xi_{1}=0
\end{array}\right. \\
& \Psi^{3} \equiv d_{3} \Phi^{2}-d_{2} \Phi^{3}+\frac{1}{x^{2}} \Phi^{3}=0, \\
& \Psi^{2} \equiv d_{3} \Phi^{1}-d_{1} \Phi^{3}=0, \\
& \Psi^{1} \equiv d_{2} \Phi^{1}-d_{1} \Phi^{2}-\frac{1}{x^{2}} \Phi^{1}=0 \\
& \Rightarrow d_{3} \Psi^{1}-d_{2} \Psi^{2}+d_{1} \Psi^{3}+\frac{1}{x^{2}} \Psi^{2}=0 \\
& \begin{array}{llll}
0 & 0 & 0 & 0 \\
\downarrow & \downarrow & \downarrow & \downarrow
\end{array}
\end{aligned}
$$

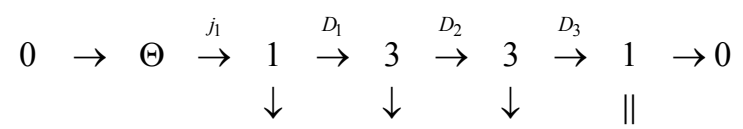

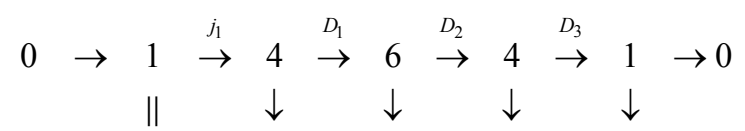

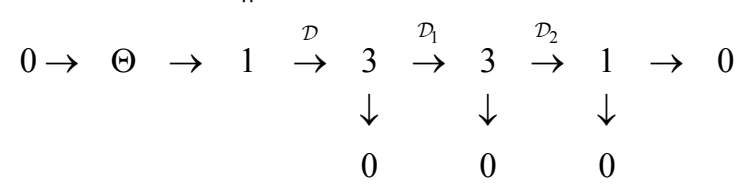

This result definitively proves that, as far as differential sequences are concerned, the only important object is the group, not the metric.

\section{Conclusion}

We may summarize the results obtained in the 3 previous subsections by saying:

$$
\text { JANET AND SPENCER PLAY AT SEE - SAW }
$$

because we have the formula $\operatorname{dim}\left(C_{r}\right)+\operatorname{dim}\left(F_{r}\right)=\operatorname{dim}\left(C_{r}(E)\right)$ and the sum thus only depends on $(n, m, q)$ but not on the underlying group when $E=T$. Hence, the smaller the background group is, the smaller the dimensions of the Spencer bundles are and the higher the dimensions of the Janet bundles are. As a byproduct, we claim that the only solution for escaping is to increase the dimension of the Lie group involved, adding successively 1 dilatation and 4 elations in order to deal with the conformal group of space-time while using the Spencer sequence instead of the Janet sequence ([23] [43]). The results of this paper thus question the mathematical foundations of GR and even strengthen the doubts we already had about the existence of gravitational waves in [23]. Two forthcoming publications will achieve this game. 


\section{Conflicts of Interest}

The authors declare no conflicts of interest regarding the publication of this paper.

\section{References}

[1] Andersson, L. (2015) Spin Geometry and Conservation Laws in the Kerr Spacetime. arxiv:1504.02069.

[2] Shah, A.G., Whitting, B.F., Aksteiner, S., Andersson, L. and Bäckdahl, T. (2016) Gauge Invariant Perturbation of Schwarzschild Spacetime. arxiv:1611.08291.

[3] Aksteiner, S. and Bäckdahl, T. (2018) All Local Gauge Invariants for Perturbations of the Kerr Spacetime. arxiv:1803.05341.

[4] Khavkine, I. (2017) Journal of Geometry and Physics, 113, 131-169. arxiv:1409.7212. https://doi.org/10.1016/j.geomphys.2016.06.009

[5] Khavkine, I. (2018) Compatibility Complexes of Overdetermined PDEs of Finite Type, with Applications to the Killing Equation. arxiv:1805.03751.

[6] Eisenhart, L.P. (1926) Riemannian Geometry. Princeton University Press, Princeton

[7] Vessiot, E. (1903) Annales Scientifiques de P École Normale Supérieure, 20, 411-451. (Can Be Obtained from http://numdam.org)

[8] Vessiot, E. (1904) Annales Scientifiques de PÉcole Normale Supérieure, 21, 9-85. (Can Be Obtained from http://numdam.org)

[9] Goldschmidt, H. (1968) Annales Scientifiques de lÉcole Normale Supérieure, 4, 417-444. https://doi.org/10.24033/asens.1168

[10] Goldschmidt, H. (1968) Annales Scientifiques de lÉcole Normale Supérieure, 4, 617-625. https://doi.org/10.24033/asens.1173

[11] Spencer, D.C. (1965) Bulletin of the American Mathematical Society, 75, 1-114.

[12] Pommaret, J.-F. (1978) Systems of Partial Differential Equations and Lie Pseudogroups. Gordon and Breach, New York; Russian Translation: MIR, Moscow.

[13] Pommaret, J.-F. (1983) Differential Galois Theory. Gordon and Breach, New York.

[14] Pommaret, J.-F. (1988) Lie Pseudogroups and Mechanics. Gordon and Breach, New York.

[15] Pommaret, J.-F. (1994) Partial Differential Equations and Group Theory. Kluwer, Dordrecht. https://doi.org/10.1007/978-94-017-2539-2

[16] Pommaret, J.-F. (2001) Partial Differential Control Theory. Kluwer, Dordrecht, $1000 \mathrm{p}$.

[17] Pommaret, J.-F. (2016) Deformation Theory of Algebraic and Geometric Structures. Lambert Academic Publisher, Saarbrucken. http://arxiv.org/abs/1207.1964

[18] Pommaret, J.-F. (2018) New Mathematical Methods for Physics. NOVA Science Publisher, New York.

[19] Pommaret, J.-F.(1995) Comptes rendus de I Académie des Sciences, 320, 1225-1230.

[20] Pommaret, J.-F. (2013) Multidimensional Systems and Signal Processing, 26, 405-437.

[21] Pommaret, J.-F. (2013) Journal of Modern Physics, 4, 223-239. https://doi.org/10.4236/jmp.2013.48A022

[22] Pommaret, J.-F. (2014) Journal of Modern Physics, 5, 157-170. https://doi.org/10.4236/jmp.2014.55026 
[23] Pommaret, J.-F.(2017) Journal of Modern Physics, 8, 2122-2158. https://doi.org/10.4236/jmp.2017.813130

[24] Pommaret, J.-F. (2017) Algebraic Analysis and Mathematical Physics. http://arxiv.org/abs/1706.04105

[25] Pommaret, J.-F. (2018) From Elasticity to Electromagnetism: Beyond the Mirror. http://arxiv.org/abs/1802.02430

[26] Janet, M. (1920) Journal d Analyse Mathematique, 8, 65-151.

[27] Assem, I. (1997) Algèbres et Modules. Masson, Paris.

[28] Bourbaki, N. (1980) Algébre. Ch. 10, Algébre Homologique, Masson, Paris.

[29] Hu, S.-T. (1968) Introduction to Homological Algebra, Holden-Day.

[30] Kunz, E. (1985) Introduction to Commutative Algebra and Algebraic Geometry. Birkhäuser, Boston.

[31] Northcott, D.G. (1966) An Introduction to Homological Algebra. Cambridge University Press, Cambridge.

[32] Rotman, J.J. (1979) An Introduction to Homological Algebra, Pure and Applied Mathematics. Academic Press, Cambridge.

[33] Macaulay, F.S. (1916) The Algebraic Theory of Modular Systems. Cambridge.

[34] Zerz, E. (2000) Topics in Multidimensional Linear Systems Theory. Lecture Notes in Control and Information Sciences (LNCIS) 256, Springer, Berlin.

[35] Bjork, J.E. (1993) Analytic D-Modules and Applications. Kluwer, Dordrecht. https://doi.org/10.1007/978-94-017-0717-6

[36] Quadrat, A. (2010) Les cours du CIRM, Journees Nationales de Calcul Formel, 1, 281-471.

[37] Schneiders, J.-P. (1994) Bulletin de la Societe Royale des Sciences de Liege, 63, 223-295.

[38] Quadrat, A. and Robertz, R. (2014) Acta Applicandae Mathematicae, 133, 187-234. http://hal-supelec.archives-ouvertes.fr/hal-00925533 https://doi.org/10.1007/s10440-013-9864-x

[39] Kashiwara, M. (1995) Algebraic Study of Systems of Partial Differential Equations. Mémoires de la Société Mathématique de France 63 (Transl. from Japanese of His 1970 Master's Thesis).

[40] Foster, J. and Nightingale, J.D. (1979) A Short Course in General Relativity. Longman, London.

[41] Weyl, H. (1918) Space, Time, Matter. Springer, Dover.

[42] Choquet-Bruhat, Y. (2015) Introduction to General Relativity, Black Holes and Cosmology. Oxford University Press, Oxford.

[43] Hughston, L.P. and Tod, K.P. (1990) An Introduction to General Relativity. London Math. Soc. Students Texts 5, Cambridge University Press, Cambridge. 